\title{
A COMPACTNESS TOOL FOR THE ANALYSIS OF NONLOCAL EVOLUTION EQUATIONS
}

\author{
LIVIU I. IGNAT, TATIANA I. IGNAT, DENISA STANCU-DUMITRU
}

\begin{abstract}
In this paper we give a new compactness criterion in the Lebesgue spaces $L^{p}((0, T) \times \Omega)$ and use it to obtain the first term in the asymptotic behaviour of the solutions of a nonlocal convection diffusion equation. We use previous results of Bourgain, Brezis and Mironescu to give a new criterion in the spirit of the Aubin-Lions-Simon Lemma.
\end{abstract}

\section{INTRODUCTION}

The aim of this paper is to give a new version of the classical compactness arguments in the space $L^{p}((0, T) \times \Omega)$, [29], one which can be adapted to nonlocal evolution equations. We will apply this new criterion for the analysis of the long time behavior of the solutions of the following system

$$
\left\{\begin{array}{l}
u_{t}=J * u-u+G *|u|^{q-1} u-|u|^{q-1} u, \quad x \in \mathbb{R}^{d}, t>0 \\
u(0)=\varphi
\end{array}\right.
$$

where $J$ and $G$ are non-negative $L^{1}(\mathbb{R})$-functions with mass one having a second and first momentum respectively. More details about the assumptions on these two functions will be given later.

Let us now recall a classical compactness result in the spaces $L^{p}((0, T), B)$, with $B$ a Banach space. Aubin-Lions-Simon Lemma [29, Th. 5] assumes that we have three Banach spaces $X \hookrightarrow B \hookrightarrow Y$ where the embedding $X \hookrightarrow B$ is compact. A sequence $\left\{f_{n}\right\}_{n \geq 1}$ is relatively compact in $L^{p}((0, T), B)$ (and in $C([0, T], B)$ if $\left.p=\infty\right)$ if we can guarantee that $\left\{f_{n}\right\}_{n \geq 1}$ is bounded in $L^{p}((0, T), X)$ and $\left\|\tau_{h} f_{n}-f_{n}\right\|_{L^{p}((0, T-h), Y)} \rightarrow 0$ as $h \rightarrow 0$ uniformly in $n$.

There are situations where we cannot bound uniformly a sequence $\left\{g_{n}\right\}_{n \geq 1}$ in a space that is compactly embedded in $L^{p}(\Omega)$. Instead of that we have estimates on some Dirichlet forms that vary with $n$, estimates that allow us to obtain the compactness of the sequence $\left\{g_{n}\right\}_{n \geq 1}$ (see for example [3], [24] and [2, Th. 6.11, p. 128]). Let us now be more precise. We choose $1 \leq p<\infty$ and $\Omega \subset \mathbb{R}^{d}$ a smooth domain. Function $\rho: \mathbb{R}^{d} \rightarrow \mathbb{R}$ is a nonnegative smooth radial function with compact support, non identically zero, satisfying $\rho(x) \geq \rho(y)$

Key words and phrases. Nonlocal diffusion, compactness arguments, convection-diffusion, asymptotic behaviour

2000 Mathematics Subject Classification. 35B40, 45G10, 46B50. 
if $|x| \leq|y|$. Set $\rho_{n}(x)=n^{d} \rho(n x)$. Let $\left\{g_{n}\right\}_{n \geq 1}$ be a bounded sequence in $L^{p}(\Omega)$ such that

$$
n^{p} \int_{\Omega} \int_{\Omega} \rho_{n}(x-y)\left|g_{n}(x)-g_{n}(y)\right|^{p} d x d y \leq M .
$$

Then as proved in [3], [24], [2, Th. 6.11, p. 128], sequence $\left\{g_{n}\right\}_{n \geq 1}$ is relatively compact in $L^{p}(\Omega)$. Our main contribution is to use this compactness argument instead of the compact embedding $X \hookrightarrow B$ in the Aubin-Lions-Simon Lemma and to obtain a new compactness criterion in $L^{p}((0, T) \times \Omega)$.

The main compactness tool that we prove in this paper is the following one.

Theorem 1.1. Let $1 \leq p<\infty$ and $\Omega \subset \mathbb{R}^{d}$ be an open set. Let $\rho: \mathbb{R}^{d} \rightarrow \mathbb{R}$ be a nonnegative smooth radial function with compact support, non identically zero, and $\rho_{n}(x)=n^{d} \rho(n x)$. Let $\left\{f_{n}\right\}_{n \geq 1}$ be a sequence of functions in $L^{p}((0, T) \times \Omega)$ such that

$$
\int_{0}^{T} \int_{\Omega}\left|f_{n}(t, x)\right|^{p} d x d t \leq M
$$

and

$$
n^{p} \int_{0}^{T} \int_{\Omega} \int_{\Omega} \rho_{n}(x-y)\left|f_{n}(t, x)-f_{n}(t, y)\right|^{p} d x d y d t \leq M
$$

1. If $\left\{f_{n}\right\}_{n \geq 1}$ is weakly convergent in $L^{p}((0, T) \times \Omega)$ to $f$ then $f \in L^{p}\left((0, T), W^{1, p}(\Omega)\right)$ for $p>1$ and $f \in L^{1}((0, T), B V(\Omega))$ for $p=1$.

2. Let $p>1$. Assuming that $\Omega$ is a smooth bounded domain in $\mathbb{R}^{d}, \rho(x) \geq \rho(y)$ if $|x| \leq|y|$ and that

$$
\left\|\partial_{t} f_{n}\right\|_{L^{p}\left((0, T), W^{-1, p}(\Omega)\right)} \leq M
$$

then $\left\{f_{n}\right\}_{n \geq 1}$ is relatively compact in $L^{p}((0, T) \times \Omega)$.

Extensions to mixed type space norms of the type $L^{p}\left((0, T), L^{q}(\Omega)\right)$ could also be obtained by adapting the estimates in this paper. The possibility of obtaining more general nonlocal compactness tools as in Aubin-Lions-Simon Lemma (see Theorem 2.2 below) remains to be analyzed. In (1.4) for technical reasons we considered the space $W^{-1, p}(\Omega)$ but we believe that the results still hold by replacing $W^{-1, p}(\Omega)$ with any space $Y$ such that $L^{p}(\Omega) \hookrightarrow Y$ continuously. More general functions $\rho_{n}$ can be considered, like those in [24] but this is beyond the scope of this article. We leave to the reader the possible extension of the above result to the case when $\rho_{n}$ are not necessarily obtained by rescaling a given function $\rho$. The case of general weights $\rho_{n}$ will introduce new difficulties since technical results in Lemma 2.2 and Lemma 2.4, used in the proof of Theorem 1.1. make use in an essential manner of the fact that the weights are obtained by scaling from a given function $\rho$.

Once we prove Theorem 1.1 we apply it in the analysis of the asymptotic behaviour of system (1.1). Recently, similar results to those in Theorem 1.1 have been employed in the analysis of some numerical splitting methods for Burgers like equations [13].

Let us now be more precise about the assumptions on the kernels $J$ and $G$. We assume that $J, G: \mathbb{R}^{d} \rightarrow \mathbb{R}$ are non-negative functions satisfying the following assumptions 
(H0) $\int_{\mathbb{R}^{d}} J(z) d z=\int_{\mathbb{R}^{d}} G(z) d z=1$,

(H1) $J \in L^{1}\left(\mathbb{R}^{d}, 1+|x|^{2}\right), G \in L^{1}\left(\mathbb{R}^{d}, 1+|x|\right)$,

(H2) $J$ is positive in a neighborhood of the origin

(H3) $J$ is symmetric, i.e. $J(z)=J(-z)$. In particular the first moment of $J$ vanishes

$$
\int_{\mathbb{R}^{d}} J(z) z_{j} d z=0, \quad j=1, \ldots, d
$$

Condition (H0) is assumed for simplicity. In fact the same analysis can be done for integral equations of the type

$$
\left\{\begin{array}{l}
u_{t}(t, x)=\int_{\mathbb{R}^{d}} J(x-y)(u(t, y)-u(t, x)) d y+ \\
\quad+\int_{\mathbb{R}^{d}} G(x-y)\left(|u|^{q-1} u(t, y)-|u|^{q-1} u(t, x)\right) d y, \quad x \in \mathbb{R}^{d}, t>0, \\
u(0)=\varphi,
\end{array}\right.
$$

without assuming that the mass of each of the functions $J$ and $G$ is one.

The well-posedness of this model has been analyzed in [14, Th. 1.1] under smoothness assumptions of the functions $J$ and $G$. We emphasize that the same global well-posedness result holds under the assumptions that $J$ and $G$ are non-negative $L^{1}\left(\mathbb{R}^{d}\right)$-functions: for any $q>1$ and $\varphi \in L^{1}\left(\mathbb{R}^{d}\right) \cap L^{\infty}\left(\mathbb{R}^{d}\right)$ there exists a unique global solution $u \in C\left([0, \infty), L^{1}\left(\mathbb{R}^{d}\right) \cap\right.$ $\left.L^{\infty}\left(\mathbb{R}^{d}\right)\right)$ satisfying

$$
\|u(t)\|_{L^{1}\left(\mathbb{R}^{d}\right)} \leq\|\varphi\|_{L^{1}\left(\mathbb{R}^{d}\right)} \quad \text { and } \quad\|u(t)\|_{L^{\infty}\left(\mathbb{R}^{d}\right)} \leq\|\varphi\|_{L^{\infty}\left(\mathbb{R}^{d}\right)} .
$$

Since $J$ and $G$ have mass one the mass conservation property holds

$$
\int_{\mathbb{R}^{d}} u(t, x) d x=\int_{\mathbb{R}^{d}} \varphi(x) d x .
$$

Since the proof of the global well-posedness follows by the same fix point argument as in [14] we will omit it here.

Observe that under the assumptions (H0), (H1) and (H3) there exist positive constants $R, \delta$ such that

$$
\left\{\begin{array}{cc}
\widehat{J}(\xi) \leq 1-\frac{A}{2}|\xi|^{2}, & |\xi| \leq R, \\
\widehat{J}(\xi) \leq 1-\delta, & |\xi| \geq R,
\end{array}\right.
$$

where $A=\frac{1}{2} \int_{\mathbb{R}^{d}} J(z)|z|^{2} d z$. Under these assumptions it has been proved in [14, Th. 1.4] that the solutions of (1.1) decay similar to the classical heat equation: for any $1 \leq p<\infty$ the following holds:

$$
\|u(t)\|_{L^{p}\left(\mathbb{R}^{d}\right)} \leq C\left(p, d,\|\varphi\|_{L^{1}\left(\mathbb{R}^{d}\right)},\|\varphi\|_{L^{\infty}\left(\mathbb{R}^{d}\right)}\right)(t+1)^{-\frac{d}{2}\left(1-\frac{1}{p}\right)} .
$$


This decay property has been obtained in [14] by the so-called Fourier Splitting method [25, 26, 27] and in a more general setting in [16]. When $p=2$ a similar argument has also been used in [28]. As far as the authors know, the case $p=\infty$ in (1.5) is open.

In the case when the nonlinear term is supercritical, i.e. $q>1+1 / d$, the first term in the asymptotic behavior has been analyzed in 14 under the additional assumption that $J \in \mathcal{S}\left(\mathbb{R}^{d}\right)$, the class of rapidly decreasing functions. There the main idea was that the nonlinear part decays faster than the linear semigroup and then the first term in the long time behavior is given by the linear semigroup. This has been already observed in [8] in the case of the classical convection-diffusion system.

The aim of this paper is to give an answer to the critical case $q=1+1 / d$ even though we give a proof that both treats the critical and super-critical case. We emphasize that we do not require function $J$ to belong to $\mathcal{S}\left(\mathbb{R}^{d}\right)$ as in [14]. The subcritical case $1<q<1+1 / d$ is still open. The method we employ is the so-called four step method that consists in the analysis of some rescaled orbits $\left\{u_{\lambda}(t)\right\}$. We refer to 32 for a review of the method in the case of the porous medium equation.

We consider two important quantities

$$
A=\frac{1}{2} \int_{\mathbb{R}^{d}} J(z)|z|^{2} d z \quad \text { and } \quad B=\left(B_{1}, \ldots, B_{d}\right), B_{j}=\int_{\mathbb{R}^{d}} G(z) z_{j} d z, j=1, \ldots, d .
$$

The main result concerning system (1.1) is the following one.

Theorem 1.2. Let $J$ and $G$ be two non-negative functions satisfying hypotheses (HO)-(H3). For any $\varphi \in L^{1}\left(\mathbb{R}^{d}\right) \cap L^{\infty}\left(\mathbb{R}^{d}\right)$ the solution $u$ of system (1.1) satisfies

$$
\lim _{t \rightarrow \infty} t^{\frac{d}{2}\left(1-\frac{1}{p}\right)}\left\|u(t)-t^{-d / 2} f_{m}\left(\frac{x}{\sqrt{t}}\right)\right\|_{L^{p}\left(\mathbb{R}^{d}\right)}=0, \quad 1 \leq p<\infty,
$$

where the profile $f_{m}$ is the smooth solution of the equation

$$
-A \Delta f_{m}-\frac{1}{2} x \cdot \nabla f_{m}=\frac{d}{2} f_{m}-\alpha B \cdot \nabla\left(\left|f_{m}\right|^{q-1} f_{m}\right) \quad \text { in } \mathbb{R}^{d},
$$

with $\int_{\mathbb{R}^{d}} f_{m}=m$ where $m$ is the mass of the initial data $\varphi$ and

$$
\alpha=\left\{\begin{array}{cc}
1, & q=1+\frac{1}{d} \\
0, & q>1+\frac{1}{d} .
\end{array}\right.
$$

Next, we say a few words about the above asymptotic profile

$$
U(t, x)=t^{-d / 2} f_{m}\left(\frac{x}{\sqrt{t}}\right) .
$$

When $q>1+1 / d$ or $B=0_{1, d}$ the asymptotic profile is the rescaled heat kernel solution of

$$
\left\{\begin{array}{l}
U_{t}=A \Delta U, \quad x \in \mathbb{R}^{d}, t>0 \\
U(0)=m \delta_{0}
\end{array}\right.
$$


When $q=1+1 / d$ and $B \neq 0_{1, d}, U$ is the unique solution of the following equation

$$
\left\{\begin{array}{l}
U_{t}=A \Delta U-B \cdot \nabla\left(|U|^{1 / d} U\right), \quad x \in \mathbb{R}^{d}, t>0 \\
U(0)=m \delta_{0}
\end{array}\right.
$$

The well-posedness of this system has been analyzed in [9] in the one-dimensional case and in [10] in the multi-dimensional case. It has been proved in [1] that the profile $f_{m}$ is of constant sign and decays exponentially to zero as $|x| \rightarrow \infty$.

We remark that in the case of the symmetric function $G$, i.e. $G(z)=G(-z)$, the solution of (1.1) converges to the heat kernel since in this case $B$ vanishes. When $B \neq 0$ we obtain in the limit the solutions of the viscous convection-diffusion equation. Along the paper we will consider the case of nonnegative initial data, so nonnegative solutions of system (1.1). The case of sign-change solutions could also be analyzed with small modifications of the proof (see [9] for a rigorous treatment of the critical case for the convection-diffusion equation).

In the linear case, i.e. $u_{t}=J * u-u$, the asymptotic behavior has been obtained in [5] by means of Fourier analysis techniques and in [18] by scaling methods. In [18] the scaling argument works since it is applied to the smooth part of the solution. Refined asymptotics have been obtained in [15, 17]. We also recall here [30, 31] where a scaling method is used for equations of the type $u_{t}=J * u-u-u^{p}$. There the authors obtain barriers for $W$ and its derivatives, $W$ being the smooth part of the solution of the linear equation $u_{t}=J * u-u$. Once these barriers are obtained the authors split the solution of the nonlinear problem in a way that permits obtaining uniform Hölder estimates and then compactness. The method developed here is more flexible in the sense that it uses only energy estimates that involve the linear part of the equation and the good sign of the nonlinearity.

In the local case, i.e. $u_{t}=\Delta u+a \cdot \nabla\left(|u|^{q-1} u\right)$, the same analysis has been performed in a series of papers. In [8] the case $q \geq 1+1 / d$ is treated and the results in the critical case have been obtained by a careful space-time change of variables and using weighted Sobolev spaces. The sub-critical case is more difficult and the one-dimensional case has been considered in 9]. The extension to higher dimensions has been obtained in [10] and [4].

In contrast with the analysis in [8] here we assume that the initial data belong to the space $L^{1}\left(\mathbb{R}^{d}\right) \cap L^{\infty}\left(\mathbb{R}^{d}\right)$. This assumption is necessary since even in the linear case $u_{t}=J * u-u$ a lack of smoothing effect is present. More precisely the solutions of the linear model are as regular as the initial data. In the case of the heat equation with initial data in $L^{1}\left(\mathbb{R}^{d}\right)$ the solution at any positive time belongs to any $L^{p}\left(\mathbb{R}^{d}\right)$ space $1 \leq p \leq \infty$, and this type of gain of integrability can also be proved for the nonlinear convection-diffusion 8 .

We recall some similar models to those analyzed here. In [22] the author considers a one-dimensional model that is nonlocal in the diffusive part $u_{t}=J * u-u+u u_{x}$ with $J=e^{-|x|}$ and he proves that its solutions converge to the ones of Bourger's equation with Dirac delta initial data. However the key tool used there, an Oleinik estimate $\partial_{x} u(t) \leq 1 / t$ in $\mathcal{D}^{\prime}(\mathbb{R})$ is not available in our model. The methods used here can be adapted to analyze 
similar models but with nonlinearities of the type $\left(u^{q}\right)_{x}, q \geq 2$, [12]. In these cases, entropy conditions in the sense of Kružkov [21] should be imposed on weak solutions in order to have a well-posed problem. This does not appear in our model since the nonlinearity does not involve derivatives.

The models considered here could be related with the ones considered in [7] where a scalar conservation law with a diffusion-type source of the type $u_{t}+\nabla \cdot f(u)=\Delta P_{s} u$ is analyzed. There $P_{s}$ is essentially given by $\widehat{P_{s} u}(\xi) \simeq\left(1+|\xi|^{2}\right)^{-s} \hat{u}(\xi)$ and even more general models are considered. However, in order to obtain the long time behavior of the solutions, the authors assume that the initial data belong to some $H^{N}\left(\mathbb{R}^{d}\right)$ spaces where $N$ is large enough. In view of our analysis here we believe that the results in [7] can be obtained by assuming less regularity on the initial data. The analysis of these models by our methods remains to be considered in future papers.

Similar nonlocal models have been introduced recently in [6] where the nonlocal convective term takes the form

$$
\int_{\mathbb{R}} \phi_{0}(y-x)\left(\frac{u(t, y)+u(t, x)}{2}\right)^{2} d y
$$

where $\phi_{0}$ is an odd function. The possible application of the methods introduced here remains to be analyzed. From the very beginning, the main difficulty in these models is the global existence of the solutions. Some models when the convection is nonlocal have been considered previously in [19], $u_{t}=u_{x x}+G * u^{q}-u^{q}, q \geq 2$ and [20], $u_{t}=$ $u_{x x}+\left(u^{q-1}(K * u)\right)_{x}, q=2$. The main difficulty in obtaining the asymptotic behavior for similar models where the convection is dominant, i.e. $1<q<2$, is to obtain an entropy estimate. Even if the entropy inequality can be avoided in the critical case it seems to be crucial for the uniqueness of the solutions of the limit equation in the sub-critical case. However, we refer to [4] where the asymptotic behavior of systems of the type $u_{t}=$ $\Delta u-\partial_{y}\left(|u|^{q-1} u\right)$ with $q$ subcritical is obtained without entropy estimates but rather with a kinetic formulation that allows to use some compactness arguments previously employed in the case of multidimensional scalar conservation laws [23]. The possible application of these kinetic methods to the case on nonlocal diffusion and/or convection remains to be analyzed in the future.

The paper is organized as follows. In Section 2 we review a few compactness arguments known to be useful in the analysis of time evolution problems and prove Theorem 1.1. Once the compactness tool is obtained, in Section 3 we prove Theorem 1.2 ,

\section{Compactness Tools}

In this section we review a few classical compactness tools and give some results that will allow us to prove the main result of this paper.

First we recall some results given in [29] about the characterization of compact sets in $L^{p}((0, T), B)$ where $B$ is a Banach space and $1 \leq p \leq \infty$.

Theorem 2.1 (29], Th. 1). Let $\mathcal{F} \subset L^{p}((0, T), B) . \mathcal{F}$ is relatively compact in $L^{p}((0, T), B)$ for $1 \leq p<\infty$, or $C([0, T], B)$ for $p=\infty$ if and only if 
(1) $\left\{\int_{t_{1}}^{t_{2}} f(t) d t, f \in \mathcal{F}\right\}$ is relatively compact in $B$ for all $0<t_{1}<t_{2}<T$,

(2) $\left\|\tau_{h} f-f\right\|_{L^{p}((0, T-h), B)} \rightarrow 0$ as $h \rightarrow 0$ uniformly for $f \in \mathcal{F}$.

The following criterion is also given.

Theorem 2.2 ([29], Th. 5). Let us consider three Banach spaces $X \hookrightarrow B \hookrightarrow Y$ where $X \hookrightarrow B$ is compact. Assume $1 \leq p \leq \infty$ and

i) $\mathcal{F}$ is bounded in $L^{p}((0, T), X)$,

ii) $\left\|\tau_{h} f-f\right\|_{L^{p}((0, T-h), Y)} \rightarrow 0$ as $h \rightarrow 0$ uniformly for $f \in \mathcal{F}$.

Then $\mathcal{F}$ is relatively compact in $L^{p}((0, T), B)$ (and in $C([0, T], B)$ if $\left.p=\infty\right)$.

The last criterion is obtained mainly by using Theorem 2.1 and the following inequality that follows from the fact that $X$ is compactly embedded in $B$ : for any $\varepsilon>0$ there exists $\eta(\varepsilon)>0$ such that

$$
\|u\|_{B} \leq \varepsilon\|u\|_{X}+\eta(\varepsilon)\|u\|_{Y}, \quad \forall u \in X
$$

In the nonlocal setting we will obtain a similar inequality in Lemma 2.2.

Now we recall some compactness results that have been proved in the nonlocal context [3], 2] and in a more general setting in [24].

Theorem 2.3 ([2], Th. 6.11, p. 128). Let $1 \leq p<\infty$ and $\Omega \subset \mathbb{R}^{d}$ be an open set. Let $\rho: \mathbb{R}^{d} \rightarrow \mathbb{R}^{d}$ be a nonnegative smooth radial function with compact support, non identically zero, and $\rho_{n}(x)=n^{d} \rho(n x)$. Let $\left\{f_{n}\right\}_{n \geq 1}$ be a bounded sequence in $L^{p}(\Omega)$ such that

$$
n^{p} \int_{\Omega} \int_{\Omega} \rho_{n}(x-y)\left|f_{n}(x)-f_{n}(y)\right|^{p} d x d y \leq M .
$$

The following hold:

1. If $\left\{f_{n}\right\}_{n \geq 1}$ is weakly convergent in $L^{p}(\Omega)$ to $f$ then $f \in W^{1, p}(\Omega)$ for $p>1$ and $f \in B V(\Omega)$ for $p=1$. Moreover

$$
\|\nabla f\|_{L^{p}(\Omega)} \leq C(\Omega, \rho) M
$$

2. Assuming that $\Omega$ is a smooth bounded domain in $\mathbb{R}^{d}$ and $\rho(x) \geq \rho(y)$ if $|x| \leq|y|$ then $\left\{f_{n}\right\}_{n \geq 1}$ is relatively compact in $L^{p}(\Omega)$.

We point out that the assumption on the compact support of function $\rho$ could be removed. In fact once we have estimate (2.2) for a function $\rho$ we also have this estimate for any compactly supported function $\tilde{\rho}$ with $\tilde{\rho} \leq \rho$.

The above results hold under more general assumptions on the weights $\left\{\rho_{n}\right\}_{n \geq 1}$ and on a bounded domain $\Omega$ in $\mathbb{R}^{d}$ with Lipshitz boundary. As proved in [24, Th. 1.2] we can 
assume that $\left\{\tilde{\rho}_{n}\right\}_{n \geq 1}$ is a sequence of radially symmetric functions in $L^{1}\left(\mathbb{R}^{d}\right)$ satisfying

$$
\left\{\begin{array}{l}
\tilde{\rho}_{n} \geq 0, \text { a.e. in } \mathbb{R}^{d}, \\
\int_{\mathbb{R}^{d}} \tilde{\rho}_{n}(x)=1, \forall n \geq 1, \\
\lim _{n \rightarrow \infty} \int_{|x|>\delta} \tilde{\rho}_{n}(x) d x=0, \quad \forall \delta>0
\end{array}\right.
$$

and that

$$
\int_{\Omega} \int_{\Omega} \frac{\tilde{\rho}_{n}(x-y)}{|x-y|^{p}}\left|f_{n}(x)-f_{n}(y)\right|^{p} d x d y \leq M .
$$

Then the results in Theorem 2.3 remain true in dimension $d \geq 2$. In dimension $d=1$ some technical assumptions have to be assumed [24, Th. 1.3]. Choosing $\tilde{\rho}_{n}(x)=n^{d}(n|x|)^{p} \rho(n x)$ with $\rho$ radial and decreasing, these technical assumptions hold and we obtain the results in the second part of Theorem 2.3. We also recall that under the above conditions on $\tilde{\rho}_{n}$ a Poincaré inequality holds [24, Th. 1.1]

$$
\left\|f_{n}-\frac{1}{|\Omega|} \int_{\Omega} f_{n}\right\|_{L^{p}(\Omega)}^{p} \leq C\left(p, \Omega,\left\{\rho_{n}\right\}\right) \int_{\Omega} \int_{\Omega} \frac{\tilde{\rho}_{n}(x-y)}{|x-y|^{p}}\left|f_{n}(x)-f_{n}(y)\right|^{p} d x d y .
$$

In view of this inequality the boundedness of $\left\{f_{n}\right\}_{n \geq 1}$ in $L^{p}(\Omega)$ is guaranteed by (2.2) if we assume that $\left\{f_{n}\right\}_{n \geq 1}$ is bounded in $L^{1}(\Omega)$ and $\Omega$ has finite measure.

Proof of Theorem 1.1. Using the same arguments as in the proof of Theorem 2.3 (see [2, Ch. 6, p. 128]) we obtain the results in the first part.

We now prove the second part of the theorem by following the ideas in [29] but taking into account the particular estimate (1.3). From now on, in order to simplify the presentation, we assume that $\rho$ is supported in the unit ball.

Step. I. Compactness in $L^{p}\left((0, T), W^{-1, p}(\Omega)\right)$. We now check the hypotheses in Theorem 2.1. Let us choose $0 \leq t_{1}<t_{2} \leq T$ and set

$$
g_{n}(x)=\int_{t_{1}}^{t_{2}} f_{n}(s, x) d s .
$$

Estimate (1.3) gives us that

$$
n^{p} \int_{\Omega} \int_{\Omega} \rho_{n}(x-y)\left|g_{n}(x)-g_{n}(y)\right|^{p} d x d y \leq M T^{p-1} .
$$

Theorem 2.3 applied to sequence $\left\{g_{n}\right\}_{n \geq 1}$ shows that there exists $g \in W^{1, p}(\Omega)$ such that, up to a subsequence, $g_{n} \rightarrow g$ in $L^{p}(\Omega)$ so in $W^{-1, p}(\Omega)$. Estimate (1.4) shows that the second requirement in Theorem 2.1 is also satisfied. Hence $\left\{f_{n}\right\}_{n \geq 1}$ is relatively compact in $L^{p}\left((0, T), W^{-1, p}(\Omega)\right)$.

Step. II. Compactness in $L^{p}\left((0, T), L_{l o c}^{p}(\Omega)\right)$. Since $\left\{f_{n}\right\}_{n \geq 1}$ is bounded in $L^{p}((0, T) \times$ $\Omega$ ) then up to a subsequence $\left\{f_{n}\right\}_{n \geq 1}$ weakly converges to some function $f$ in $L^{p}((0, T) \times \Omega)$. The first part of Theorem 1.1 guarantees that $f \in L^{p}\left((0, T), W^{1, p}(\Omega)\right)$. 
We now use the strong convergence in $L^{p}\left((0, T), W^{-1, p}(\Omega)\right)$ obtained in Step I, estimate (1.3) and the fact that $f \in L^{p}\left((0, T), W^{1, p}(\Omega)\right)$ to prove that up to a subsequence, $\left\{f_{n}\right\}_{n \geq 1}$ strongly convergences to $f$ in $L^{p}((0, T) \times \Omega)$.

To simplify the presentation we will always denote the subsequence by $\left\{f_{n}\right\}_{n \geq 1}$. Also, when possible, we will not write all the constants in inequalities of the type $f \leq C g$ using instead $f \lesssim g$.

In the following we prove that for any $\Omega^{\prime} \subset \Omega$ such that $d\left(\Omega^{\prime}, \partial \Omega\right)>0,\left\{f_{n}\right\}_{n \geq 1}$ is relatively compact in $L^{p}\left((0, T) \times \Omega^{\prime}\right)$. From now on for a set $\mathcal{O}$ we will denote

$$
\mathcal{O}_{\rho}=\mathcal{O}+\operatorname{supp}(\rho)=\{x+\theta, x \in \mathcal{O}, \theta \in \operatorname{supp}(\rho)\}
$$

The following two Lemmas will be very useful in our analysis. Their proof will be given later.

Lemma 2.1. Let $\Omega$ be and open set of $\mathbb{R}^{d}$. For any $1<p<\infty$ there exists a positive constant $C(\rho, \Omega, p)$ such that the following inequality

$$
n^{p} \int_{\Omega} \int_{\Omega} \rho_{n}(x-y)|u(x)-u(y)|^{p} d x d y \leq C(\rho, \Omega, p) \int_{\Omega}|\nabla u|^{p}
$$

holds for all $n>0$ and $u \in W^{1, p}(\Omega)$.

Lemma 2.2. Let $\Omega$ be a bounded domain and $\chi \in C_{c}^{1}(\Omega)$. There exists a positive constant $C=C(\Omega, \chi, \rho, p)$ such that for every $\varepsilon \in(0,1)$ the following inequality

$$
C \int_{\Omega}|\chi u|^{p} \leq \varepsilon n^{p} \int_{\Omega_{\rho_{n}}} \int_{\Omega_{\rho_{n}}} \rho_{n}(x-y)|u(x)-u(y)|^{p} d x d y+\varepsilon \int_{\Omega_{\rho_{n}}}|u|^{p}+\frac{1}{\varepsilon}\|u\|_{W^{-1, p}(\Omega)}^{p}
$$

holds for all $n \varepsilon^{1 / p} \gtrsim 1$ and for all $u \in L^{p}\left(\mathbb{R}^{d}\right)$.

Remark 1. In the right hand side of inequality (2.4) we have $\varepsilon^{-1}$ and the $W^{-1, p}(\Omega)$-norm. We believe that some improvement in (2.4) can be done by allowing the norm of the last term to be in some space $Y$ with $L^{p}(\Omega) \hookrightarrow Y$ and replacing $\varepsilon^{-1}$ correspondingly. The extension of Lemma 2.2 to general spaces $Y$ will enlarge the class of nonlocal problems where the scaling arguments used in this paper can be applied.

Let us fix $\Omega^{\prime} \subset \Omega$ such that $d\left(\Omega^{\prime}, \partial \Omega\right)>0$ and choose a smooth function $\chi$ compactly supported in $\Omega$ such that $\chi \equiv 1$ in $\Omega^{\prime}$. We choose $N_{0}$ large enough such that $\Omega_{\rho_{n}}^{\prime} \subset \Omega$ for all $n \geq N_{0}$.

Applying Lemma 2.2 with $g=f_{n}-f$ to the set $\Omega^{\prime}$ we have for any $n \gtrsim \varepsilon^{-1 / p}$ that

$$
\|\chi g\|_{L^{p}\left(\Omega^{\prime}\right)}^{p} \lesssim \varepsilon n^{p} \int_{\Omega_{\rho_{n}}^{\prime}} \int_{\Omega_{\rho_{n}}^{\prime}} \rho_{n}(x-y)|g(x)-g(y)|^{p} d x d y+\varepsilon \int_{\Omega_{\rho_{n}}^{\prime}}|g|^{p}+\frac{1}{\varepsilon}\|g\|_{W^{-1, p}\left(\Omega^{\prime}\right)}^{p} .
$$


We integrate the above inequality on the time interval $[0, T]$ and use that $\|g\|_{W^{-1, p}\left(\Omega^{\prime}\right)} \leq$ $\|g\|_{W^{-1, p}(\Omega)}$ to obtain

$$
\begin{aligned}
\int_{0}^{T} \int_{\Omega^{\prime}} \chi^{p}\left|f_{n}-f\right|^{p} \lesssim & \varepsilon n^{p} \int_{0}^{T} \int_{\Omega_{\rho_{n}}^{\prime}} \int_{\Omega_{\rho_{n}}^{\prime}} \rho_{n}(x-y)\left|\left(f_{n}-f\right)(t, x)-\left(f_{n}-f\right)(t, y)\right|^{p} d x d y d t \\
& +\varepsilon \int_{0}^{T} \int_{\Omega_{\rho_{n}}^{\prime}}\left|f_{n}-f\right|^{p}+\frac{1}{\varepsilon} \int_{0}^{T}\left\|f_{n}-f\right\|_{W^{-1, p}(\Omega)}^{p} \\
\lesssim & \varepsilon n^{p} \int_{0}^{T} \int_{\Omega_{\rho_{n}}^{\prime}} \int_{\Omega_{\rho_{n}}^{\prime}} \rho_{n}(x-y)\left|f_{n}(t, x)-f_{n}(t, y)\right|^{p} d x d y d t \\
& +\varepsilon n^{p} \int_{0}^{T} \int_{\Omega_{\rho_{n}}^{\prime}} \int_{\Omega_{\rho_{n}}^{\prime}} \rho_{n}(x-y)|f(t, x)-f(t, y)|^{p} d x d y d t \\
& +\varepsilon\left(\left\|f_{n}\right\|_{L^{p}\left((0, T) \times \Omega_{\rho_{n}}^{\prime}\right)}^{p}+\|f\|_{L^{p}\left((0, T) \times \Omega_{\rho_{n}}^{\prime}\right)}^{p}\right)+\frac{1}{\varepsilon} \int_{0}^{T}\left\|f_{n}(t)-f(t)\right\|_{W^{-1, p}(\Omega)}^{p}
\end{aligned}
$$

Since for $n \gtrsim \max \left\{N_{0}, \varepsilon^{-1 / p}\right\}$ we have that $\Omega_{\rho_{n}}^{\prime} \subset \Omega$ we use estimates (1.2), (1.3), Lemma 2.1 and the fact that $f \in L^{p}\left((0, T), W^{1, p}(\Omega)\right)$ to obtain that

$$
\int_{0}^{T} \int_{\Omega^{\prime}} \chi^{p}\left|f_{n}-f\right|^{p} \lesssim \varepsilon\left(M+\|f\|_{L^{p}\left((0, T), W^{1, p}(\Omega)\right)}^{p}\right)+\frac{1}{\varepsilon} \int_{0}^{T}\left\|f_{n}(t)-f(t)\right\|_{W^{-1, p}(\Omega)}^{p} d t .
$$

Using Step I, up to a subsequence, we obtain that for any $\varepsilon \in(0,1)$

$$
\limsup _{n \rightarrow \infty} \int_{0}^{T} \int_{\Omega^{\prime}}\left|f_{n}-f\right|^{p} d x d t \lesssim \varepsilon\left(M+\|f\|_{L^{p}\left((0, T), W^{1, p}(\Omega)\right)}^{p}\right) .
$$

Then $f_{n}$ strongly converges to $f$ in $L^{p}\left((0, T) \times \Omega^{\prime}\right)$. Applying a standard diagonalisation procedure we can extract a subsequence, denoted again by $\left\{f_{n}\right\}_{n \geq 1}$, such that $f_{n} \rightarrow f$ in $L^{p}\left((0, T), L_{l o c}^{p}(\Omega)\right)$.

Step. III. Compactness in $L^{p}\left((0, T), L^{p}(\Omega)\right)$. We now use the following result in [24, Lemma 5.1, Lemma 7.2]. For a positive number $r>0$ we set

$$
\Omega_{r}:=\{x \in \Omega: d(x, \partial \Omega)>r\} .
$$

Lemma 2.3. Let $\Omega$ be a bounded Lipschitz domain of $\mathbb{R}^{d}$. There exist constants $r_{0}>0$ depending on $\Omega$ and on $\rho$ and $C_{1}, C_{2}$ (depending on $p, \Omega$ and $d$ ) so that the following holds: given $0<r<r_{0}$ we can find $N_{0} \geq 1$ such that

$$
\int_{\Omega}|g|^{p} \leq C_{1} \int_{\Omega_{r}}|g|^{p}+C_{2} r^{p} n^{p} \int_{\Omega} \int_{\Omega} \rho_{n}(x-y)|g(x)-g(y)|^{p} d x d y
$$

for every $g \in L^{p}(\Omega)$ and $n \geq N_{0}$. 
We apply the above Lemma with $g=f_{n}-f$ and integrate the resulted inequality on the time interval $(0, T)$. Thus

$$
\begin{aligned}
\int_{0}^{T} \int_{\Omega} \mid & f_{n}-\left.f\right|^{p} \\
& \lesssim \int_{0}^{T} \int_{\Omega_{r}}\left|f_{n}-f\right|^{p}+r^{p} n^{p} \int_{0}^{T} \int_{\Omega} \int_{\Omega} \rho_{n}(x-y)\left|\left(f_{n}-f\right)(t, x)-\left(f_{n}-f\right)(t, y)\right|^{p} d x d y d t \\
& \lesssim \int_{0}^{T} \int_{\Omega_{r}}\left|f_{n}-f\right|^{p}+r^{p} n^{p} \int_{0}^{T} \int_{\Omega} \int_{\Omega} \rho_{n}(x-y)\left|f_{n}(t, x)-f_{n}(t, y)\right|^{p} d x d y d t \\
& +r^{p} n^{p} \int_{0}^{T} \int_{\Omega} \int_{\Omega} \rho_{n}(x-y)|f(t, x)-f(t, y)|^{p} d x d y d t .
\end{aligned}
$$

Using estimate (1.3) and Lemma 2.1 we get

$$
\int_{0}^{T} \int_{\Omega}\left|f_{n}-f\right|^{p} \lesssim \int_{0}^{T} \int_{\Omega_{r}}\left|f_{n}-f\right|^{p}+r^{p} M+r^{p} \int_{0}^{T} \int_{\Omega}|\nabla f|^{p} .
$$

Since $f_{n} \rightarrow f$ in $L^{p}\left((0, T), L_{l o c}^{p}(\Omega)\right)$ we can let $n \rightarrow \infty$ and then for any $r \in\left(0, r_{0}\right)$ we have

$$
\limsup _{n \rightarrow \infty} \int_{0}^{T} \int_{\Omega}\left|f_{n}-f\right|^{p} \lesssim r^{p}\left(M+\int_{0}^{T} \int_{\Omega}|\nabla f|^{p}\right) .
$$

This implies that, up to a subsequence, $f_{n} \rightarrow f$ in $L^{p}\left((0, T), L^{p}(\Omega)\right)$ and the proof of Theorem 1.1 is now finished.

Proof of Lemma 2.1. We first consider the case when $\Omega=\mathbb{R}^{d}$. By scaling, it is sufficient to consider the case $n=1$. Since

$$
u(x)-u(y)=\int_{0}^{1}(x-y) \cdot \nabla u(y+s(x-y)) d s
$$

we get that

$$
\begin{aligned}
\iint_{\mathbb{R}^{2 d}} \rho(x-y)|u(x)-u(y)|^{p} d x d y & \leq \iint_{\mathbb{R}^{2 d}} \rho(x-y)|x-y|^{p} \int_{0}^{1}|\nabla u(y+s(x-y))|^{p} d s d x d y \\
& =\int_{\mathbb{R}^{d}} \rho(z)|z|^{p} \int_{\mathbb{R}^{d}}|\nabla u|^{p} .
\end{aligned}
$$

In the case of a bounded domain $\Omega$ we first extend $u$ to $\mathbb{R}^{d}$ such that $\|\nabla u\|_{L^{p}\left(\mathbb{R}^{d}\right)} \leq$ $C(\Omega)\|\nabla u\|_{L^{p}(\Omega)}$. Then we have

$$
\begin{gathered}
n^{p} \iint_{\Omega \times \Omega} \rho_{n}(x-y)|u(x)-u(y)|^{p} d x d y \leq n^{p} \iint_{\mathbb{R}^{2 d}} \rho_{n}(x-y)|u(x)-u(y)|^{p} d x d y \\
\leq C(\rho, p) \int_{\mathbb{R}^{d}}|\nabla u|^{p} \leq C(\rho, \Omega, p) \int_{\Omega}|\nabla u|^{p} .
\end{gathered}
$$

The proof of Lemma 2.1 is now complete. 
The rest of this subsection is devoted to the proof of Lemma 2.2. In order to give its proof we need some auxiliary Lemmas.

Lemma 2.4. Let $1<p<\infty$. There exists a positive constant $C=C(\rho, p, d)$ such that for every $\varepsilon \in(0,1)$ the following inequality

$$
C\|u\|_{L^{p}\left(\mathbb{R}^{d}\right)}^{p} \leq \varepsilon\left[n^{p} \int_{\mathbb{R}^{d}} \int_{\mathbb{R}^{d}} \rho_{n}(x-y)|u(x)-u(y)|^{p} d x d y+\|u\|_{L^{p}\left(\mathbb{R}^{d}\right)}^{p}\right]+\varepsilon^{-1}\|u\|_{W^{-1, p}\left(\mathbb{R}^{d}\right)}^{p}
$$

holds for all $n \varepsilon^{1 / p} \gtrsim 1$ and for all $u \in L^{p}\left(\mathbb{R}^{d}\right)$.

Before starting the proof of this Lemma a few comments are needed. The case $p=2$ is reduced after using the Fourier transform to the following inequality

$$
C(\rho) \leq \varepsilon\left[n^{2}\left(\widehat{\rho}(0)-\widehat{\rho}\left(\frac{\xi}{n}\right)\right)+1\right]+\frac{1}{\varepsilon\left(1+|\xi|^{2}\right)}, \quad \forall \xi \in \mathbb{R}^{d} .
$$

Using that $\rho$ is a smooth radially symmetric function we obtain that its Fourier transform decays at infinity and moreover, $\widehat{\rho}(0)-\widehat{\rho}(\xi) \simeq|\xi|^{2}$ for $\xi \simeq 0$. This shows the existence of two positive constants $c_{1}$ and $c_{2}$ such that

$$
\frac{c_{1}|\xi|^{2}}{1+|\xi|^{2}} \leq \widehat{\rho}(0)-\widehat{\rho}(\xi) \leq \frac{c_{2}|\xi|^{2}}{1+|\xi|^{2}}, \forall \xi \in \mathbb{R}^{d} .
$$

This property implies that inequality (2.9) holds for all $n \gtrsim \varepsilon^{-1 / 2}$.

The local version of inequality (2.8) is the following one

$$
\begin{aligned}
\|u\|_{L^{p}\left(\mathbb{R}^{d}\right)}^{p} & \lesssim \varepsilon\|u\|_{W^{1, p}\left(\mathbb{R}^{d}\right)}^{p}+\varepsilon^{-1}\|u\|_{W^{-1, p}\left(\mathbb{R}^{d}\right)}^{p} \\
& =\varepsilon\left\|(I-\Delta)^{1 / 2} u\right\|_{L^{p}\left(\mathbb{R}^{d}\right)}^{p}+\varepsilon^{-1}\left\|(I-\Delta)^{-1 / 2} u\right\|_{L^{p}\left(\mathbb{R}^{d}\right)}^{p} .
\end{aligned}
$$

We remark that when $p \neq 2$ this inequality is not a consequence of a duality argument since the dual of $W^{1, p}\left(\mathbb{R}^{d}\right)$ is $W^{-1, p^{\prime}}\left(\mathbb{R}^{d}\right)$. Inequality (2.11) holds by proving that, depending on the Fourier localization of $u$, its $L^{p}$-norm is controlled by one of the two terms in the right hand side of (2.11). In fact, for any $0<\beta<1$ using classical multiplier arguments (see [11, Ch. 5]) we have

$$
\|u\|_{L^{p}\left(\mathbb{R}^{d}\right)} \lesssim \beta\left\|(I-\Delta)^{1 / 2} u\right\|_{L^{p}\left(\mathbb{R}^{d}\right)}, \quad \operatorname{supp} \widehat{u} \subset\left\{\xi:|\xi| \gtrsim \beta^{-1}\right\}
$$

and

$$
\|u\|_{L^{p}\left(\mathbb{R}^{d}\right)} \lesssim \beta^{-1}\left\|(I-\Delta)^{-1 / 2} u\right\|_{L^{p}\left(\mathbb{R}^{d}\right)}, \quad \operatorname{supp} \widehat{u} \subset\left\{\xi:|\xi| \lesssim \beta^{-1}\right\} .
$$

Proof of Lemma 2.4. Let us first make a change of variable to avoid the presence of $\rho_{n}(x)=$ $n^{d} \rho(n x)$. Estimate (2.8) is equivalent to the following one

$$
\begin{gathered}
C\|u\|_{L^{p}(\mathbb{R})}^{p} \leq \varepsilon\left[n^{p} \int_{\mathbb{R}^{d}} \int_{\mathbb{R}^{d}} \rho(x-y)|u(x)-u(y)|^{p} d x d y+\|u\|_{L^{p}\left(\mathbb{R}^{d}\right)}^{p}\right] \\
+\varepsilon^{-1}\left\|\left(I-n^{2} \Delta\right)^{-1 / 2} u\right\|_{L^{p}\left(\mathbb{R}^{d}\right)^{p}}^{p}
\end{gathered}
$$


We use a decomposition of $u$ that has already been used in [16]. Let us choose $\eta \in C_{c}^{\infty}\left(\mathbb{R}^{d}\right)$ with

$$
\int_{\mathbb{R}^{d}} \eta=1 \quad \text { and } \quad|\nabla \eta|+|\eta| \lesssim \rho
$$

This choice of $\eta$ can be always done if $\rho$ is positive in some open set. We write

$$
u=v+w, \quad v=\eta * u, \quad w=u-v .
$$

We now emphasize some important properties of $v$ and $w$. First of all observe that both of them have the $L^{p}$-norm controlled by the $L^{p}$-norm of $u$ :

$$
\|v\|_{L^{p}\left(\mathbb{R}^{d}\right)} \leq C(\eta)\|u\|_{L^{p}\left(\mathbb{R}^{d}\right)}, \quad\|w\|_{L^{p}\left(\mathbb{R}^{d}\right)} \leq C(\eta)\|u\|_{L^{p}\left(\mathbb{R}^{d}\right)}
$$

and moreover

$$
\|u\|_{L^{p}\left(\mathbb{R}^{d}\right)} \leq\|v\|_{L^{p}\left(\mathbb{R}^{d}\right)}+\|w\|_{L^{p}\left(\mathbb{R}^{d}\right)} .
$$

Since the mass of $\eta$ is one we have the following representation for $w$ :

$$
w(x)=\int_{\mathbb{R}^{d}} \eta(x-y)(u(x)-u(y)) d y .
$$

Hölder's inequality gives us that

$$
\begin{aligned}
\int_{\mathbb{R}^{d}}|w|^{p} & \leq\left(\int_{\mathbb{R}^{d}}|\eta|\right)^{p / p^{\prime}} \int_{\mathbb{R}^{d}} \int_{\mathbb{R}^{d}}|\eta(x-y)||u(x)-u(y)|^{p} d x d y \\
& \leq C(\eta, \rho) \int_{\mathbb{R}^{d}} \int_{\mathbb{R}^{d}} \rho(x-y)|u(x)-u(y)|^{p} d x d y .
\end{aligned}
$$

In the case of $v$, since $\int_{\mathbb{R}^{d}} \partial_{x_{j}} \eta=0, j=1, \ldots, d$, we write its gradient as

$$
(\nabla v)(x)=(\nabla \eta * u)(x)=\int_{\mathbb{R}^{d}} \nabla \eta(x-y)(u(x)-u(y)) d y .
$$

Thus the same argument as before gives us that

$$
\begin{aligned}
\int_{\mathbb{R}^{d}}|\nabla v|^{p} & \leq C(\eta) \int_{\mathbb{R}^{d}} \int_{\mathbb{R}^{d}}|\nabla \eta(x-y)||u(x)-u(y)|^{p} d x d y \\
& \leq C(\eta, \rho) \int_{\mathbb{R}^{d}} \int_{\mathbb{R}^{d}} \rho(x-y)|u(x)-u(y)|^{p} d x d y .
\end{aligned}
$$

We now prove estimate (2.14). In view of (2.16) for $\varepsilon n^{p} \gtrsim 1$ we have that

$$
\int_{\mathbb{R}^{d}}|w|^{p} \lesssim \varepsilon n^{p} \int_{\mathbb{R}^{d}} \int_{\mathbb{R}^{d}} \rho(x-y)|u(x)-u(y)|^{p} d x d y .
$$

We claim that $v$ satisfies the following inequality for all $\varepsilon \in(0,1)$ and for all $n \geq 1$

$$
\|v\|_{L^{p}\left(\mathbb{R}^{d}\right)} \lesssim \varepsilon^{1 / p}\left\|\left(I-n^{2} \Delta\right)^{1 / 2} v\right\|_{L^{p}\left(\mathbb{R}^{d}\right)}+\varepsilon^{-1 / p}\left\|\left(I-n^{2} \Delta\right)^{-1 / 2} v\right\|_{L^{p}\left(\mathbb{R}^{d}\right)} .
$$


Estimates (2.19), (2.15) and (2.17) imply that

$$
\begin{aligned}
\|v\|_{L^{p}\left(\mathbb{R}^{d}\right)}^{p} \lesssim \varepsilon\left[\int_{\mathbb{R}^{d}}|v|^{p}+n^{p} \int_{\mathbb{R}^{d}}|\nabla v|^{p}\right]+\varepsilon^{-1}\left\|\left(I-n^{2} \Delta\right)^{-1 / 2} v\right\|_{L^{p}\left(\mathbb{R}^{d}\right)}^{p} \\
\quad \lesssim \varepsilon\left[\int_{\mathbb{R}^{d}}|u|^{p}+n^{p} \int_{\mathbb{R}^{d}} \int_{\mathbb{R}^{d}} \rho(x-y)|u(x)-u(y)|^{p} d x d y\right]+\varepsilon^{-1}\left\|\left(I-n^{2} \Delta\right)^{-1 / 2}(\eta * u)\right\|_{L^{p}\left(\mathbb{R}^{d}\right)}^{p} \\
\quad \lesssim \varepsilon\left[\int_{\mathbb{R}^{d}}|u|^{p}+n^{p} \int_{\mathbb{R}^{d}} \int_{\mathbb{R}^{d}} \rho(x-y)|u(x)-u(y)|^{p} d x d y\right]+\varepsilon^{-1}\left\|\left(I-n^{2} \Delta\right)^{-1 / 2} u\right\|_{L^{p}\left(\mathbb{R}^{d}\right)^{\prime}}^{p}
\end{aligned}
$$

Taking into account the above estimate and estimate (2.18) for $w$, we obtain that (2.14) holds. It remains to prove that (2.19) holds. Writing explicitly the terms in the right hand side of (2.19) we reduce it to the case $n=1$. In this case inequality (2.19) follows from estimates (2.12) and (2.13).

Lemma 2.5. Let $\Omega$ be a smooth bounded domain of $\mathbb{R}^{d}$ and $p \in(1, \infty)$. For any smooth function $\chi$ supported in $\Omega$ there exists a positive constant $C(\chi)$ such that

$$
\|\chi u\|_{W^{-1, p}\left(\mathbb{R}^{d}\right)} \leq C(\chi)\|u\|_{W^{-1, p}(\Omega)} .
$$

Proof. We consider the case of the smooth function $u$. The general case follows by density. By the definition of the space $W^{-1, p}\left(\mathbb{R}^{d}\right)$ there exists a sequence $\varphi_{n} \in W^{1, p^{\prime}}\left(\mathbb{R}^{d}\right)$ with $\left\|\varphi_{n}\right\|_{W^{1, p^{\prime}\left(\mathbb{R}^{d}\right)} \leq 1 \text { such that }}$

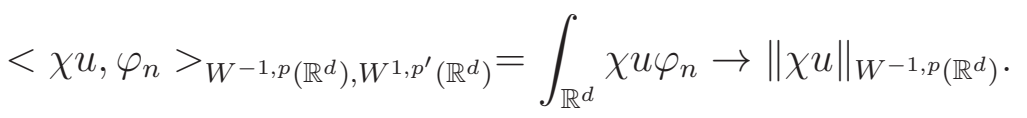

Since $\chi$ has the support included in $\Omega$, we have $\chi \varphi_{n} \in W_{0}^{1, p^{\prime}}(\Omega)$ and

$$
\left\|\chi \varphi_{n}\right\|_{W_{0}^{1, p^{\prime}}(\Omega)} \leq\|\chi\|_{W^{1, \infty}(\Omega)}\left\|\varphi_{n}\right\|_{W^{1, p^{\prime}\left(\mathbb{R}^{d}\right)}} \leq C(\chi) .
$$

Hence

$$
\int_{\mathbb{R}^{d}} \chi u \varphi_{n} \leq\|u\|_{W^{-1, p}(\Omega)}\left\|\chi \varphi_{n}\right\|_{W_{0}^{1, p^{\prime}}(\Omega)} \leq C(\chi)\|u\|_{W^{-1, p}(\Omega)}
$$

Letting $n \rightarrow \infty$ we obtain the desired estimate.

Lemma 2.6. Let $\rho: \mathbb{R}^{d} \rightarrow \mathbb{R}$ be a radial function with compact support, $\rho(0) \neq 0$, $\Omega$ be a domain in $\mathbb{R}^{d}$ and $1<p<\infty$. For any smooth function $\chi$ supported in $\Omega$ there exists a positive constant $C=C(\chi, p, \Omega)$ such that the following inequality

$$
\begin{aligned}
C n^{p} & \int_{\mathbb{R}^{d}} \int_{\mathbb{R}^{d}} \rho_{n}(x-y)|(\chi u)(x)-(\chi u)(y)|^{p} d x d y \\
& \leq n^{p} \int_{\Omega_{\rho_{n}}} \int_{\Omega_{\rho_{n}}} \rho_{n}(x-y)|u(x)-u(y)|^{p} d x d y+\left(\int_{\mathbb{R}^{d}} \rho(z)|z|^{p}\right) \int_{\Omega_{\rho_{n}}}|u|^{p} .
\end{aligned}
$$

holds for any $n>0$ and any $u \in L^{p}\left(\mathbb{R}^{d}\right)$.

Proof. Let us first observe that since $\rho$ is radially symmetric and $\rho(0) \neq 0$ we have

$$
\operatorname{supp}\left(\rho_{n}\right)=\frac{1}{n} \operatorname{supp}(\rho) .
$$


For $x \notin \Omega_{\rho_{n}}$ and $y \in \Omega$ we have that $\rho_{n}(x-y)=0$. If $y \notin \Omega$ then $\chi(x)=\chi(y)=0$. Similar things hold if we interchange $x$ and $y$. Hence

$$
\begin{aligned}
n^{p} \int_{\mathbb{R}^{d}} \int_{\mathbb{R}^{d}} \rho_{n}(x-y)|(\chi u)(x)-(\chi u)(y)|^{p} d x d y & \\
& =n^{p} \int_{\Omega_{\rho_{n}}} \int_{\Omega_{\rho_{n}}} \rho_{n}(x-y)|(\chi u)(x)-(\chi u)(y)|^{p} d x d y .
\end{aligned}
$$

Using the following identity

$$
(\chi u)(x)-(\chi u)(y)=\chi(x)(u(x)-u(y))+u(y)(\chi(x)-\chi(y))
$$

we obtain that

$$
\begin{aligned}
n^{p} \int_{\Omega_{\rho_{n}}} \int_{\Omega_{\rho_{n}}} \rho_{n}(x-y)|(\chi u)(x)-(\chi u)(y)|^{p} d x d y \\
\quad \lesssim n^{p}\|\chi\|_{L^{\infty}(\Omega)}^{p} \int_{\Omega_{\rho_{n}}} \int_{\Omega_{\rho_{n}}} \rho_{n}(x-y)|u(x)-u(y)|^{p} d x d y \\
\quad+n^{p} \int_{\Omega_{\rho_{n}}} \int_{\Omega_{\rho_{n}}} \rho_{n}(x-y)|u(y)|^{p}|\chi(x)-\chi(y)|^{p} d x d y .
\end{aligned}
$$

Using identity (2.7) for $\chi$ it follows that

$$
\begin{aligned}
n^{p} \int_{\Omega_{\rho_{n}}} & \int_{\Omega_{\rho_{n}}} \rho_{n}(x-y)|u(y)|^{p}|\chi(x)-\chi(y)|^{p} d x d y \\
& \leq n^{p} \int_{\Omega_{\rho_{n}}} \int_{\Omega_{\rho_{n}}} \rho_{n}(x-y)|u(y)|^{p}|x-y|^{p} \int_{0}^{1}|(\nabla \chi)(y+s(x-y))|^{p} d s d x d y \\
& \leq\|\chi\|_{W^{1, \infty}\left(\mathbb{R}^{d}\right)} \int_{\mathbb{R}^{d}} \rho(z)|z|^{p} d z \int_{\Omega_{\rho_{n}}}|u(y)|^{p} d y .
\end{aligned}
$$

Putting together estimates (2.22), (2.23) and (2.24) we infer the desired estimate (2.21).

Proof of Lemma 2.2. From Lemma 2.4 we know that for any $\varepsilon \in(0,1)$ and $n \varepsilon^{1 / p} \gtrsim 1$ the following inequality holds for all $v \in L^{p}\left(\mathbb{R}^{d}\right)$ :

$$
\|v\|_{L^{p}\left(\mathbb{R}^{d}\right)}^{p} \lesssim \varepsilon n^{p} \int_{\mathbb{R}^{d}} \int_{\mathbb{R}^{d}} \rho_{n}(x-y)|v(x)-v(y)|^{p} d x d y+\frac{1}{\varepsilon}\|v\|_{W^{-1, p}\left(\mathbb{R}^{d}\right)}^{p}+\varepsilon\|v\|_{L^{p}\left(\mathbb{R}^{d}\right)}^{p} .
$$

We now localize the above inequality by applying it to $v=\chi u$ where $\chi$ has been extended by zero outside of $\Omega$. Thus

$$
\int_{\Omega}|\chi u|^{p} \lesssim \varepsilon n^{p} \int_{\mathbb{R}^{d}} \int_{\mathbb{R}^{d}} \rho_{n}(x-y)|(\chi u)(x)-(\chi u)(y)|^{p} d x d y+\frac{1}{\varepsilon}\|\chi u\|_{W^{-1, p\left(\mathbb{R}^{d}\right)}}^{p}+\varepsilon\|\chi u\|_{L^{p}\left(\mathbb{R}^{d}\right)}^{p} .
$$

By Lemma 2.5 and Lemma 2.6 we deduce that

$$
\int_{\Omega}|\chi u|^{p} \lesssim \varepsilon n^{p} \int_{\Omega_{\rho_{n}}} \int_{\Omega_{\rho_{n}}} \rho_{n}(x-y)|u(x)-u(y)|^{p} d x d y+\varepsilon \int_{\Omega_{\rho_{n}}}|u|^{p}+\frac{1}{\varepsilon}\|u\|_{W^{-1, p}(\Omega)}^{p}
$$

and the proof is finished. 


\section{Proof of Theorem 1.2}

Before starting the proof of Theorem 1.2 we need some preliminary results that will be used throughout the proof.

3.1. Preliminaries. In the following we denote

$$
J_{\lambda}(x)=\lambda^{d} J(\lambda x), G_{\lambda}(x)=\lambda^{d} G(\lambda x), \widetilde{G}(x)=G(-x), \widetilde{G}_{\lambda}(x)=\lambda^{d} \widetilde{G}(\lambda x) .
$$

Lemma 3.1. The following integration by parts identities hold

$$
\begin{aligned}
\int_{\mathbb{R}^{d}}(J * \Phi-\Phi)(x) \Psi(x) d x=\int_{\mathbb{R}^{d}} \Phi(x)(J * \Psi-\Psi)(x) d x \\
=-\frac{1}{2} \int_{\mathbb{R}^{d}} \int_{\mathbb{R}^{d}} J(x-y)(\Phi(x)-\Phi(y))(\Psi(x)-\Psi(y)) d x d y
\end{aligned}
$$

and

$$
\int_{\mathbb{R}^{d}}(G * \Phi-\Phi)(x) \Psi(x) d x=\int_{\mathbb{R}^{d}} \Phi(x)(\widetilde{G} * \Psi-\Psi)(x) d x .
$$

Proof. Use Fubini's theorem and in the first case the fact that $J(-z)=J(z)$.

Lemma 3.2. For any $p \in[1, \infty]$ there exist two positive constants $C(p, J)$ and $C(p, G)$ such that

$$
\left\|\lambda^{2}\left(J_{\lambda} * \psi-\psi\right)\right\|_{L^{p}\left(\mathbb{R}^{d}\right)} \leq C(p, J)\left\|D^{2} \psi\right\|_{L^{p}\left(\mathbb{R}^{d}\right)}
$$

and

$$
\left\|\lambda\left(\widetilde{G}_{\lambda} * \psi-\psi\right)\right\|_{L^{p}\left(\mathbb{R}^{d}\right)} \leq C(p, G)\|\nabla \psi\|_{L^{p}\left(\mathbb{R}^{d}\right)}
$$

hold for all $\lambda>0$ and $\psi \in C_{c}^{2}\left(\mathbb{R}^{d}\right)$.

Proof. We treat the cases $p=1$ and $p=\infty$ since the other cases follow by interpolation. A Taylor expansion up to the second order gives us that for any $x, y \in \mathbb{R}^{d}$ the following holds

$$
\psi(y)-\psi(x)=\nabla \psi(x)(y-x)+\int_{0}^{1}(1-s)(y-x) D^{2} \psi(x+s(y-x))(y-x)^{t} d s .
$$

After a change of variables we have

$$
\begin{aligned}
\lambda^{2}\left(J_{\lambda} * \psi-\psi\right)(x) & =\lambda^{d+2} \int_{\mathbb{R}^{d}} J(\lambda(x-y))(\psi(y)-\psi(x)) d y=\lambda^{2} \int_{\mathbb{R}^{d}} J(z)\left(\psi\left(x-\frac{z}{\lambda}\right)-\psi(x)\right) d z \\
& =\lambda^{2} \int_{\mathbb{R}^{d}} J(z)\left[-\frac{z}{\lambda} \cdot \nabla \psi(x)+\frac{1}{\lambda^{2}} \int_{0}^{1}(1-s) z D^{2} \psi\left(x-\frac{s z}{\lambda}\right) z^{t} d s\right] d z .
\end{aligned}
$$

Since $J$ is radially symmetric we have

$$
\int_{\mathbb{R}^{d}} J(z) z_{j} d z=0 \text { for all } j=1, \ldots, d
$$


and

$$
\int_{\mathbb{R}^{d}} J(z) z_{j} z_{k} d z=0 \quad \text { for all } 1 \leq j \neq k \leq d .
$$

Those identities give us that

$$
\lambda^{2}\left(J_{\lambda} * \psi-\psi\right)(x)=\sum_{j, k=1}^{d} \int_{0}^{1}(1-s) \int_{\mathbb{R}^{d}} J(z) z_{j} z_{k} \frac{\partial^{2} \psi}{\partial x_{j} \partial x_{k}}\left(x-\frac{s z}{\lambda}\right) d z d s
$$

and then for $p \in\{1, \infty\}$ inequality (3.3) holds with $C(J)=\frac{1}{2} \int_{\mathbb{R}^{d}} J(z)|z|^{2} d z$.

In the case of the second estimate (3.4) we use the identity:

$$
\psi(y)-\psi(x)=\int_{0}^{1}(y-x) \cdot \nabla \psi(x+s(y-x)) d s
$$

to obtain

$$
\begin{aligned}
\lambda\left(\widetilde{G}_{\lambda}\right. & * \psi-\psi)(x)=\lambda^{d+1} \int_{\mathbb{R}^{d}} \tilde{G}(\lambda(x-y))(\psi(y)-\psi(x)) d y \\
& =\lambda \int_{\mathbb{R}^{d}} \tilde{G}(z)\left(\psi\left(x-\frac{z}{\lambda}\right)-\psi(x)\right) d y=\int_{\mathbb{R}^{d}} \tilde{G}(z) \int_{0}^{1} z \cdot \nabla \psi\left(x-\frac{s z}{\lambda}\right) d s d z \\
& =\int_{\mathbb{R}^{d}} G(z) \int_{0}^{1} z \cdot \nabla \psi\left(x+\frac{s z}{\lambda}\right) d s d z .
\end{aligned}
$$

Using the same arguments as in the first case we obtain the second estimate.

3.2. Proof of Theorem 1.2. We consider the family $\left\{u_{\lambda}(t)\right\}_{\lambda>0}$ defined by

$$
u_{\lambda}(t, x)=\lambda^{d} u\left(\lambda^{2} t, \lambda x\right) .
$$

It follows that $u_{\lambda}$ is a solution of the following rescaled equation

$$
\left\{\begin{array}{l}
\left(u_{\lambda}\right)_{t}=\lambda^{2}\left(J_{\lambda} * u_{\lambda}-u_{\lambda}\right)+\lambda^{d(1-q)+2}\left(G_{\lambda} * u_{\lambda}^{q}-u_{\lambda}^{q}\right), \quad x \in \mathbb{R}^{d}, t>0, \\
u_{\lambda}(0, x)=\varphi_{\lambda}(x),
\end{array}\right.
$$

where $\varphi_{\lambda}(x)=\lambda^{d} \varphi(\lambda x)$.

The proof of Theorem 1.2 is divided into four steps.

Step I. Estimates on the rescaled solutions $u_{\lambda}$. We recall [14, Theorem 1.4] that solution $u$ of system (1.1) satisfies for any $p \in[1, \infty)$ and $t>0$ the following estimate

$$
\|u(t)\|_{L^{p}\left(\mathbb{R}^{d}\right)} \leq C\left(p,\|\varphi\|_{L^{1}\left(\mathbb{R}^{d}\right)},\|\varphi\|_{L^{\infty}\left(\mathbb{R}^{d}\right)}\right)(t+1)^{-\frac{d}{2}\left(1-\frac{1}{p}\right)} .
$$

In the sequel we will denote by $C$ a constant that may change from line to line, may depend on $\|\varphi\|_{L^{1}\left(\mathbb{R}^{d}\right)}$ and $\|\varphi\|_{L^{\infty}\left(\mathbb{R}^{d}\right)}$ but it is independent of the scaling parameter $\lambda$. In the following lemmas the constant $M$ will depend on $\|\varphi\|_{L^{1}\left(\mathbb{R}^{d}\right)}$ and $\|\varphi\|_{L^{\infty}\left(\mathbb{R}^{d}\right)}$. We will not make explicit this dependence unless this is necessary. 
Lemma 3.3. For any $0<t_{1}<t_{2}<\infty$ and $p \in[1, \infty)$ there exists a positive constant $M=M\left(t_{1}, p\right)$ such that

holds for all $\lambda>0$.

$$
\left\|u_{\lambda}\right\|_{L^{\infty}\left(\left(t_{1}, t_{2}\right), L^{p}\left(\mathbb{R}^{d}\right)\right)} \leq M
$$

Proof. Using estimate (3.10) and the fact that the rescaled solutions satisfy

$$
\left\|u_{\lambda}(t)\right\|_{L^{p}\left(\mathbb{R}^{d}\right)}=\lambda^{d\left(1-\frac{1}{p}\right)}\left\|u\left(\lambda^{2} t\right)\right\|_{L^{p}\left(\mathbb{R}^{d}\right)},
$$

we deduce that for any $p \in[1, \infty)$ and $t>0$ the following inequality holds for all $\lambda>0$ :

$$
\left\|u_{\lambda}(t)\right\|_{L^{p}\left(\mathbb{R}^{d}\right)} \leq C\left(\frac{\lambda^{2}}{\lambda^{2} t+1}\right)^{\frac{d}{2}\left(1-\frac{1}{p}\right)} \leq C t^{-\frac{d}{2}\left(1-\frac{1}{p}\right)}
$$

Using that $t \geq t_{1}$ we obtain the desired estimate.

Lemma 3.4. For any $0<t_{1}<t_{2}<\infty$ there exists a positive constant $M=M\left(t_{1}\right)$ such that the following inequality

$$
\lambda^{2} \int_{t_{1}}^{t_{2}} \int_{\mathbb{R}^{d}} \int_{\mathbb{R}^{d}} J_{\lambda}(x-y)\left(u_{\lambda}(t, x)-u_{\lambda}(t, y)\right)^{2} d x d y d t \leq M
$$

holds for all $\lambda>0$.

Proof. Multiplying (3.9) by $u_{\lambda}$ and integrating over $\mathbb{R}^{d}$ we get

$$
\frac{1}{2} \frac{d}{d t}\left\|u_{\lambda}(t)\right\|_{L^{2}\left(\mathbb{R}^{d}\right)}^{2}=\int_{\mathbb{R}^{d}} \lambda^{2}\left(J_{\lambda} * u_{\lambda}-u_{\lambda}\right) u_{\lambda}(t) d x+\int_{\mathbb{R}^{d}} \lambda^{d(1-q)+2}\left(G_{\lambda} * u_{\lambda}^{q}-u_{\lambda}^{q}\right) u_{\lambda}(t) d x .
$$

Using that $G_{\lambda}$ has mass one the last term in the above identity is negative. Indeed,

$$
\begin{aligned}
\int_{\mathbb{R}^{d}}\left(G_{\lambda} * u_{\lambda}^{q}\right)(t, x) u_{\lambda}(t, x) d x=\int_{\mathbb{R}^{d}} \int_{\mathbb{R}^{d}} G_{\lambda}(x-y) u_{\lambda}^{q}(t, y) u_{\lambda}(t, x) d x d y \\
\quad \leq \int_{\mathbb{R}^{d}} \int_{\mathbb{R}^{d}} G_{\lambda}(x-y)\left(\frac{q}{q+1} u_{\lambda}^{q+1}(t, y)+\frac{1}{q+1} u_{\lambda}^{q+1}(t, x)\right) d x d y=\int_{\mathbb{R}^{d}} u_{\lambda}^{q+1}(t, x) d x .
\end{aligned}
$$

Next, integrating (3.12) over the interval $\left(t_{1}, t_{2}\right)$ and using identity (3.1) we obtain

$$
\left\|u_{\lambda}\left(t_{2}\right)\right\|_{L^{2}\left(\mathbb{R}^{d}\right)}^{2}+\lambda^{2} \int_{t_{1}}^{t_{2}} \int_{\mathbb{R}^{d}} \int_{\mathbb{R}^{d}} J_{\lambda}(x-y)\left(u_{\lambda}(t, x)-u_{\lambda}(t, y)\right)^{2} d x d y d t \leq\left\|u_{\lambda}\left(t_{1}\right)\right\|_{L^{2}\left(\mathbb{R}^{d}\right)}^{2} .
$$

Using inequality (3.11) in the case $p=2$ we conclude that

$$
\lambda^{2} \int_{t_{1}}^{t_{2}} \int_{\mathbb{R}^{d}} \int_{\mathbb{R}^{d}} J_{\lambda}(x-y)\left(u_{\lambda}(t, x)-u_{\lambda}(t, y)\right)^{2} d x d y d t \leq C t_{1}^{-\frac{d}{2}}
$$

and the proof finishes.

Lemma 3.5. For any $0<t_{1}<t_{2}<\infty$ there exists a positive constant $M=M\left(t_{1}\right)$ such that

holds for all $\lambda>1$.

$$
\left\|u_{\lambda, t}\right\|_{L^{2}\left(\left(t_{1}, t_{2}\right), H^{-1}\left(\mathbb{R}^{d}\right)\right)} \leq M
$$


Proof. Multiplying (3.9) by $\psi \in C_{c}^{2}\left(\mathbb{R}^{d}\right)$, integrating over $\mathbb{R}^{d}$ and using Lemma 3.1 we get

$$
\begin{aligned}
\int_{\mathbb{R}^{d}} u_{\lambda, t}(t, x) \psi(x) d x= & \int_{\mathbb{R}^{d}} \lambda^{2}\left(J_{\lambda} * u_{\lambda}-u_{\lambda}\right) \psi(x) d x+\int_{\mathbb{R}^{d}} \lambda^{d(1-q)+2}\left(G_{\lambda} * u_{\lambda}^{q}-u_{\lambda}^{q}\right) \psi(x) d x \\
= & -\frac{\lambda^{2}}{2} \int_{\mathbb{R}^{d}} \int_{\mathbb{R}^{d}} J_{\lambda}(x-y)(\psi(x)-\psi(y))\left(u_{\lambda}(t, x)-u_{\lambda}(t, y)\right) d x d y \\
& +\int_{\mathbb{R}^{d}} \lambda^{d(1-q)+2}\left(\widetilde{G}_{\lambda} * \psi-\psi\right) u_{\lambda}^{q}(t, x) d x,
\end{aligned}
$$

where $\widetilde{G}_{\lambda}(x)=G_{\lambda}(-x)$. Using Cauchy's inequality, the fact that $\lambda>1$ and $q \geq 1+1 / d$ we get

$$
\begin{aligned}
\left|\int_{\mathbb{R}^{d}} u_{\lambda, t}(t, x) \psi(x) d x\right| \leq & \left(\frac{\lambda^{2}}{2} \int_{\mathbb{R}^{d}} \int_{\mathbb{R}^{d}} J_{\lambda}(x-y)(\psi(x)-\psi(y))^{2} d x d y\right)^{1 / 2} \\
& \times\left(\frac{\lambda^{2}}{2} \int_{\mathbb{R}^{d}} \int_{\mathbb{R}^{d}} J_{\lambda}(x-y)\left(u_{\lambda}(t, x)-u_{\lambda}(t, y)\right)^{2} d x d y\right)^{1 / 2} \\
& +\left\|\lambda\left(\widetilde{G}_{\lambda} * \psi-\psi\right)\right\|_{L^{2}\left(\mathbb{R}^{d}\right)}\left(\int_{\mathbb{R}^{d}}\left|u_{\lambda}(t, x)\right|^{2 q} d x\right)^{1 / 2}
\end{aligned}
$$

Applying Lemma 2.1 to $J_{\lambda}$ and $\psi$, Lemma 3.2 to $\tilde{G}_{\lambda}$ and estimate (3.11) to $u_{\lambda}$ we deduce that

$$
\begin{aligned}
& \left|\int_{\mathbb{R}^{d}} u_{\lambda, t}(t, x) \psi(x) d x\right| \\
& \quad \lesssim\|\psi\|_{H^{1}\left(\mathbb{R}^{d}\right)}\left[\left(\lambda^{2} \int_{\mathbb{R}^{d}} \int_{\mathbb{R}^{d}} J_{\lambda}(x-y)\left(u_{\lambda}(t, x)-u_{\lambda}(t, y)\right)^{2} d x d y\right)^{1 / 2}+t^{-\frac{d}{4}(2 q-1)}\right] .
\end{aligned}
$$

Thus

$$
\left\|u_{\lambda, t}(t)\right\|_{H^{-1}\left(\mathbb{R}^{d}\right)}^{2} \lesssim \lambda^{2} \int_{\mathbb{R}^{d}} \int_{\mathbb{R}^{d}} J_{\lambda}(x-y)\left(u_{\lambda}(t, x)-u_{\lambda}(t, y)\right)^{2} d x d y+t^{-\frac{d}{2}(2 q-1)} .
$$

Integrating this inequality on the time interval $\left(t_{1}, t_{2}\right)$ and then applying Lemma 3.4 we obtain the desired result.

Step II. Compactness in $L_{l o c}^{1}\left((0, \infty), L^{1}\left(\mathbb{R}^{d}\right)\right)$. We first establish the compactness of the family $\left\{u_{\lambda}\right\}$ in $L_{l o c}^{1}\left((0, \infty) \times \mathbb{R}^{d}\right)$. Using estimates on the tail of $\left\{u_{\lambda}\right\}$ we will obtain strong convergence in $L_{l o c}^{1}\left((0, \infty), L^{1}\left(\mathbb{R}^{d}\right)\right)$.

Lemma 3.3 and Lemma 3.5 give us that $\left\{u_{\lambda}\right\}$ is uniformly bounded in $L_{l o c}^{\infty}\left((0, \infty), L_{l o c}^{2}\left(\mathbb{R}^{d}\right)\right)$ and $\left\{\partial_{t} u_{\lambda}\right\}$ is uniformly bounded in $L_{l o c}^{2}\left((0, \infty), H^{-1}(\Omega)\right)$ for any bounded domain $\Omega$ of $\mathbb{R}^{d}$. Taking into account that $L^{2}(\Omega)$ is compactly embedded in $H^{-\varepsilon}(\Omega)$ for any $\varepsilon>0$, and $H^{-\varepsilon}(\Omega)$ is continuously embedded in $H^{-1}(\Omega)$ for $0<\varepsilon<1$, by classical compactness arguments ([29], Corollary 4, p. 85) we deduce that $\left\{u_{\lambda}\right\}$ is relatively compact in $C\left(\left[t_{1}, t_{2}\right], H^{-\varepsilon}(\Omega)\right)$ for all $0<t_{1}<t_{2}$ and $0<\varepsilon<1$. Extracting a subsequence we get

$$
u_{\lambda_{n}} \rightarrow U \text { in } C\left(\left[t_{1}, t_{2}\right], H^{-\varepsilon}(\Omega)\right) \text {. }
$$


Using estimate (3.11) we obtain that for each fixed $t>0$, the family $\left\{u_{\lambda_{n}}(t)\right\}_{n \geq 1}$ is uniformly bounded in $L_{l o c}^{p}\left(\mathbb{R}^{d}\right)$. Then any subsequence $\left\{u_{\lambda_{k_{n}}}(t)\right\}_{n \geq 1}$ weakly convergent should converge to $U(t)$. Indeed, if $u_{\lambda_{k_{n}}}(t) \rightarrow v$ in $L^{p}(\Omega)$ then $u_{\lambda_{k_{n}}}(t) \rightarrow v$ in $\mathcal{D}^{\prime}(\Omega)$ and hence $v=U(t)$. This fact shows that for every $t>0$ and $p \in(1, \infty)$ we have

$$
u_{\lambda_{n}}(t) \rightarrow U(t) \text { in } L_{l o c}^{p}\left(\mathbb{R}^{d}\right) .
$$

The uniform bound given in (3.11) of $\left\{u_{\lambda}(t)\right\}$ transfers to $U(t)$. Hence, the limit point $U$ belongs to $L_{l o c}^{\infty}\left((0, \infty), L^{p}\left(\mathbb{R}^{d}\right)\right)$ for all $1<p<\infty$ and moreover we get that

$$
\|U(t)\|_{L^{p}\left(\mathbb{R}^{d}\right)} \leq \liminf _{\lambda \rightarrow \infty}\left\|u_{\lambda}(t)\right\|_{L^{p}\left(\mathbb{R}^{d}\right)} \leq \frac{C}{t^{\frac{d}{2}\left(1-\frac{1}{p}\right)}}, \quad \forall t>0 .
$$

Let us now prove the strong convergence in $L_{l o c}^{1}\left((0, \infty) \times \mathbb{R}^{d}\right)$. Lemma 3.3, Lemma 3.4 and Lemma 3.5 show that for any $0<t_{1}<t_{2}<\infty$ there exists $M=M\left(t_{1},\|\varphi\|_{L^{1}\left(\mathbb{R}^{d}\right)},\|\varphi\|_{L^{\infty}\left(\mathbb{R}^{d}\right)}\right)$ such that

$$
\begin{gathered}
\left\|u_{\lambda}\right\|_{L^{2}\left(\left(t_{1}, t_{2}\right) \times \mathbb{R}^{d}\right)} \leq M, \\
\lambda^{2} \int_{t_{1}}^{t_{2}} \int_{\mathbb{R}^{d}} \int_{\mathbb{R}^{d}} J_{\lambda}(x-y)\left(u_{\lambda}(t, x)-u_{\lambda}(t, y)\right)^{2} d x d y d t \leq M
\end{gathered}
$$

and

$$
\left\|u_{\lambda, t}\right\|_{L^{2}\left(\left(t_{1}, t_{2}\right), H^{-1}\left(\mathbb{R}^{d}\right)\right)} \leq M .
$$

Let us now choose a function $\rho$ as in Theorem 1.1 such that $0 \leq \rho \leq J$. In view of hypothesis (H2) this is always possible. Also (3.16) holds with function $\rho$ instead of $J$. We apply Theorem 1.1 to family $\left\{u_{\lambda}\right\}_{\lambda>0}$ and to the time interval $\left(t_{1}, t_{2}\right)$. We obtain that there exists a function $v \in L^{2}\left(\left(t_{1}, t_{2}\right), H^{1}\left(\mathbb{R}^{d}\right)\right)$ such that, up to a subsequence,

$$
u_{\lambda} \rightarrow v \quad \text { in } L^{2}\left(\left(t_{1}, t_{2}\right) ; L_{l o c}^{2}\left(\mathbb{R}^{d}\right)\right) .
$$

The previous analysis shows that $v=U$. Thus $U \in L_{l o c}^{2}\left((0, \infty), H^{1}\left(\mathbb{R}^{d}\right)\right)$ and, up to a subsequence

$$
u_{\lambda} \rightarrow U \text { in } L_{l o c}^{1}\left((0, \infty) \times \mathbb{R}^{d}\right) .
$$

We now prove that in fact $u_{\lambda}$ strongly converges to $U$ in $L_{l o c}^{1}\left((0, \infty), L^{1}\left(\mathbb{R}^{d}\right)\right)$. Using a standard diagonal argument the compactness in $L_{l o c}^{1}\left((0, \infty), L^{1}\left(\mathbb{R}^{d}\right)\right)$ is reduced to the fact that for any $0<t_{1}<t_{2}<\infty$ the following holds

$$
\int_{t_{1}}^{t_{2}}\left\|u_{\lambda}(t)\right\|_{L^{1}(|x|>R)} d t \rightarrow 0 \quad \text { as } \quad R \rightarrow \infty \text {, uniformly in } \lambda \geq 1 .
$$

This follows from the Lemma below since the initial data $\varphi$ belongs to $L^{1}\left(\mathbb{R}^{d}\right)$.

Lemma 3.6. There exists a constant $C=C\left(J, G,\|\varphi\|_{L^{1}\left(\mathbb{R}^{d}\right)},\|\varphi\|_{L^{\infty}\left(\mathbb{R}^{d}\right)}\right)$ such that the following inequality

$$
\int_{|x|>2 R} u_{\lambda}(t, x) d x \leq \int_{|x|>R} \varphi(x) d x+C\left(\frac{t}{R^{2}}+\frac{t^{1 / 2}}{R}\right)
$$

holds for any $t>0$ and $R>0$, uniformly on $\lambda \geq 1$. 
Proof. Let $\psi \in C^{\infty}\left(\mathbb{R}^{d}\right)$ be such that $0 \leq \psi \leq 1$ and satisfies $\psi(x) \equiv 0$ for $|x|<1$ and $\psi(x) \equiv 1$ for $|x|>2$. We put $\psi_{R}(x)=\psi(x / R)$. We multiply equation (3.9) by $\psi_{R}$ and integrate by parts to obtain

$$
\begin{aligned}
\int_{\mathbb{R}^{d}} u_{\lambda}(t, x) \psi_{R}(x) d x-\int_{\mathbb{R}^{d}} \varphi_{\lambda}(x) \psi_{R}(x) d x= & \lambda^{2} \int_{0}^{t} \int_{\mathbb{R}^{d}} u_{\lambda}(s, x)\left(J_{\lambda} * \psi_{R}-\psi_{R}\right) d x d s \\
& +\lambda^{d(1-q)+2} \int_{0}^{t} \int_{\mathbb{R}^{d}} u_{\lambda}^{q}(s, x)\left(\widetilde{G}_{\lambda} * \psi_{R}-\psi_{R}\right)(x) d x d s .
\end{aligned}
$$

We now use Lemma 3.2 with $p=\infty$, the fact that

$$
\left\|D^{2}\left(\psi_{R}\right)\right\|_{L^{\infty}\left(\mathbb{R}^{d}\right)}=R^{-2}\left\|D^{2} \psi\right\|_{L^{\infty}\left(\mathbb{R}^{d}\right)}, \quad\left\|\nabla \psi_{R}\right\|_{L^{\infty}\left(\mathbb{R}^{d}\right)}=R^{-1}\|\nabla \psi\|_{L^{\infty}\left(\mathbb{R}^{d}\right)}
$$

and the conservation of the $L^{1}\left(\mathbb{R}^{d}\right)$-norm of $u_{\lambda}$ to find that

$$
\begin{aligned}
\int_{\mathbb{R}^{d}} u_{\lambda}(t, x) \psi_{R}(x) d x \lesssim & \int_{\mathbb{R}^{d}} \varphi_{\lambda}(x) \psi_{R}(x) d x+R^{-2}\left\|D^{2} \psi\right\|_{L^{\infty}\left(\mathbb{R}^{d}\right)} \int_{0}^{t} \int_{\mathbb{R}^{d}} u_{\lambda}(s, x) d x d s \\
& +\lambda^{d(1-q)+1} R^{-1}\|\nabla \psi\|_{L^{\infty}\left(\mathbb{R}^{d}\right)} \int_{0}^{t} \int_{\mathbb{R}^{d}} u_{\lambda}^{q}(s, x) d x d s \\
\lesssim & \int_{|x|>R} \varphi_{\lambda}(x) d x+R^{-2}\left\|D^{2} \psi\right\|_{L^{\infty}\left(\mathbb{R}^{d}\right)} t\|\varphi\|_{L^{1}\left(\mathbb{R}^{d}\right)} \\
& +\lambda^{d(1-q)+1} R^{-1}\|\nabla \psi\|_{L^{\infty}\left(\mathbb{R}^{d}\right)} \int_{0}^{t} \int_{\mathbb{R}^{d}} u_{\lambda}^{q}(s, x) d x d s .
\end{aligned}
$$

To estimate the last term in the above inequality we use the decay of $u_{\lambda}$ given by (3.11) and obtain that

$$
\lambda^{d(1-q)+1} \int_{0}^{t} \int_{\mathbb{R}^{d}} u_{\lambda}^{q}(s, x) d x d s \lesssim \lambda^{d(1-q)+1} \int_{0}^{t} \frac{\lambda^{d(q-1)} d s}{\left(1+\lambda^{2} s\right)^{\frac{d(q-1)}{2}}}=\lambda^{-1} \int_{0}^{t \lambda^{2}} \frac{d s}{(1+s)^{\frac{d(q-1)}{2}}} .
$$

Since

$$
\lim _{x \rightarrow \infty} x^{-1} \int_{0}^{x^{2}} \frac{d s}{(1+s)^{\frac{d(q-1)}{2}}}=\lim _{x \rightarrow \infty} \frac{2 x}{\left(1+x^{2}\right)^{\frac{d(q-1)}{2}}}= \begin{cases}0, & q>1+\frac{1}{d}, \\ 2, & q=1+\frac{1}{d},\end{cases}
$$

we find that

$$
\lambda^{d(1-q)+1} \int_{0}^{t} \int_{\mathbb{R}^{d}} u_{\lambda}^{q}(s, x) d x d s \lesssim C t^{1 / 2} .
$$

Going back to (3.20), using that $\lambda>1$ and $\psi(x) \equiv 1$ for $|x|>2$ we get

$$
\int_{|x|>2 R} u_{\lambda}(t, x) d x \leq \int_{|x|>\lambda R} \varphi(x) d x+C\left(\frac{t}{R^{2}}+\frac{t^{1 / 2}}{R}\right) \leq \int_{|x|>R} \varphi(x) d x+C\left(\frac{t}{R^{2}}+\frac{t^{1 / 2}}{R}\right)
$$

and the proof of the Lemma is finished.

In view of (3.18) $\left\{u_{\lambda}\right\}_{\lambda>0}$ is relatively compact in $L_{\text {loc }}^{1}\left((0, \infty), L^{1}\left(\mathbb{R}^{d}\right)\right)$ so $u_{\lambda} \rightarrow U$ in $L_{l o c}^{1}\left((0, \infty), L^{1}\left(\mathbb{R}^{d}\right)\right)$. This result also shows that for a.e. $t>0$ we have

$$
\left\|u_{\lambda}(t)-U(t)\right\|_{L^{1}\left(\mathbb{R}^{d}\right)} \rightarrow 0 \text { as } \lambda \rightarrow \infty .
$$


In particular $\|U(t)\|_{L^{1}\left(\mathbb{R}^{d}\right)}=m$, a.e. $t>0$.

This fact will be used in Step IV to obtain the main convergence result of this paper.

Step IIIa. Passing to the limit. Using the results obtained in the previous step we can pass to the weak limit in the equation involving $u_{\lambda}$. Let us choose $0<\tau<t$. For any test function $\psi \in C_{c}^{\infty}\left(\mathbb{R}^{d}\right)$ we multiply equation (3.9) by $\psi$ and we integrate on $(\tau, t) \times \mathbb{R}^{d}$. We get

$$
\begin{aligned}
& \int_{\mathbb{R}^{d}} u_{\lambda}(t, x) \psi(x) d x-\int_{\mathbb{R}^{d}} u_{\lambda}(\tau, x) \psi(x) d x \\
& =\int_{\tau}^{t} \int_{\mathbb{R}^{d}} \lambda^{2}\left(J_{\lambda} * u_{\lambda}-u_{\lambda}\right) \psi(x) d x d s+\lambda^{d(1-q)+2} \int_{\tau}^{t} \int_{\mathbb{R}^{d}}\left(G_{\lambda} * u_{\lambda}^{q}-u_{\lambda}^{q}\right) \psi(x) d x d s \\
& =\int_{\tau}^{t} \int_{\mathbb{R}^{d}} \lambda^{2}\left(J_{\lambda} * \psi-\psi\right) u_{\lambda}(s, x) d x d s+\lambda^{d(1-q)+2} \int_{\tau}^{t} \int_{\mathbb{R}^{d}}\left(\widetilde{G}_{\lambda} * \psi-\psi\right) u_{\lambda}^{q}(s, x) d x d s .
\end{aligned}
$$

First of all observe that since for any $t>0, u_{\lambda}(t) \rightarrow U(t)$ in $L_{l o c}^{p}\left(\mathbb{R}^{d}\right), 1<p<\infty$, we have

$$
\int_{\mathbb{R}^{d}} u_{\lambda}(t, x) \psi(x) d x-\int_{\mathbb{R}^{d}} u_{\lambda}(\tau, x) \psi(x) d x \rightarrow \int_{\mathbb{R}^{d}} U(t, x) \psi(x) d x-\int_{\mathbb{R}^{d}} U(\tau, x) \psi(x) d x .
$$

Using identity (3.7) and the Lebesgue dominated convergence theorem we obtain that

$$
\lambda^{2}\left(J_{\lambda} * \psi-\psi\right)(x) \rightarrow \frac{1}{2} \sum_{i, j=1}^{n} \int_{\mathbb{R}^{d}} J(z) z_{i} z_{j} \frac{\partial^{2} \psi}{\partial x_{i} \partial x_{j}}=A \Delta \psi(x),
$$

where

$$
A=\frac{1}{2} \int_{\mathbb{R}^{d}} J(z)|z|^{2} d z
$$

Since $u_{\lambda} \rightarrow U$ in $L^{1}\left((\tau, t) \times \mathbb{R}^{d}\right)$ we obtain by using the Lebesgue theorem that

$$
\int_{\tau}^{t} \int_{\mathbb{R}^{d}} \lambda^{2}\left(J_{\lambda} * \psi-\psi\right) u_{\lambda}(s, x) d x d s \rightarrow A \int_{\tau}^{t} \int_{\mathbb{R}^{d}} \Delta \psi U(s, x) d x d s .
$$

For the term involving $\tilde{G}$ we prove that

$$
\lambda^{d(1-q)+2} \int_{\tau}^{t} \int_{\mathbb{R}^{d}}\left(\widetilde{G}_{\lambda} * \psi-\psi\right) u_{\lambda}^{q}(s, x) d x d s \rightarrow \begin{cases}0, & q>1+\frac{1}{d}, \\ \int_{\tau}^{t} \int_{\mathbb{R}^{d}} B \cdot \nabla \psi(x) U^{q}(s, x) d x d s, & q=1+\frac{1}{d},\end{cases}
$$

where

$$
B=\left(B_{1}, \ldots, B_{d}\right), B_{j}=\int_{\mathbb{R}^{d}} G(z) z_{j} d z
$$


When $q>1+1 / d$ we use Lemma 3.2 for $\widetilde{G}$ and estimate (3.11) on the $L^{p}$-norms of $u_{\lambda}$ to get

$$
\begin{aligned}
&\left.\lambda^{d(1-q)+2} \int_{\tau}^{t} \int_{\mathbb{R}^{d}} \mid \widetilde{G}_{\lambda} * \psi-\psi\right)(x) \mid u_{\lambda}^{q}(s, x) d x d s \\
& \quad \leq \lambda^{d(1-q)+1}\left\|\lambda\left(\widetilde{G}_{\lambda} * \psi-\psi\right)\right\|_{L^{\infty}\left(\mathbb{R}^{d}\right)} \int_{\tau}^{t} \int_{\mathbb{R}^{d}} u_{\lambda}^{q}(s, x) d x d s \\
& \quad \lesssim\|\nabla \psi\|_{L^{\infty}\left(\mathbb{R}^{d}\right)} \lambda \int_{\tau}^{t} \frac{d s}{\left(1+\lambda^{2} s\right)^{\frac{d}{2}(q-1)}} \\
& \lesssim\|\nabla \psi\|_{L^{\infty}\left(\mathbb{R}^{d}\right)} \lambda^{1-d(q-1)} \rightarrow 0 \text { as } \lambda \rightarrow \infty
\end{aligned}
$$

Let us now consider the case $q=1+1 / d$. First observe that identity (3.8) and Lebesgue convergence theorem give us that for any $x \in \mathbb{R}^{d}$

$$
\lambda\left(\tilde{G}_{\lambda} * \psi-\psi\right)(x) \rightarrow B \cdot \nabla \psi(x), \quad \lambda \rightarrow \infty .
$$

The results in the previous step and Hölder's inequality give us that for a.e. $t>0$ the following holds

$$
\begin{aligned}
\left\|u_{\lambda}^{q}(t)-U^{q}(t)\right\|_{L^{1}\left(\mathbb{R}^{d}\right)} & \lesssim \int_{\mathbb{R}^{d}}\left|u_{\lambda}(t)-U(t)\right|^{1 / 2}\left(\left|u_{\lambda}(t)\right|^{q-1 / 2}+|U(t)|^{q-1 / 2}\right) d x \\
& \lesssim\left\|u_{\lambda}(t)-U(t)\right\|_{L^{1}\left(\mathbb{R}^{d}\right)}^{1 / 2}\left(\left\|u_{\lambda}(t)\right\|_{L^{2 q-1}\left(\mathbb{R}^{d}\right)}^{\frac{2 q-1}{2}}+\|U(t)\|_{L^{2 q-1}\left(\mathbb{R}^{d}\right)}^{\frac{2 q-1}{2}}\right) \\
& \lesssim C(t)\left\|u_{\lambda}(t)-U(t)\right\|_{L^{1}\left(\mathbb{R}^{d}\right)} \rightarrow 0, \quad \lambda \rightarrow \infty .
\end{aligned}
$$

Hence $u_{\lambda}^{q} \rightarrow U^{q}$ in $L^{1}\left((\tau, t), L^{1}\left(\mathbb{R}^{d}\right)\right)$ and in consequence the second convergence in (3.23) holds.

All the above convergences show that $U$ satisfies

$$
\begin{aligned}
\int_{\mathbb{R}^{d}} U & (t, x) \psi(x) d x-\int_{\mathbb{R}^{d}} U(\tau, x) \psi(x) d x \\
& =A \int_{\tau}^{t} \int_{\mathbb{R}^{d}} U(s, x) \Delta \psi(x) d x d s+\alpha \int_{\tau}^{t} \int_{\mathbb{R}^{d}} U^{q}(s, x) B \cdot \nabla \psi(x) d x d s,
\end{aligned}
$$

where $\alpha=1$ if $q=1+1 / d$ and $\alpha=0$ for $q>1+1 / d$. Thus, when $q>1+1 / d$ or $B \neq 0_{1, d}$, $U$ is a distributional solution of the heat equation $U_{t}=A \Delta U$. When $q=1+1 / d$ and $B \neq 0_{1, d}, U$ is a distributional solution of the equation: $U_{t}=A \Delta U-B \cdot \nabla\left(U^{q}\right)$.

Step IIIb. Identification of the initial data. Let us choose $\tau=0$ in the previous step. Using estimate (3.21) and the mass conservation of $u_{\lambda}$ for any $\psi \in C_{c}^{2}\left(\mathbb{R}^{d}\right)$ we get

$$
\begin{aligned}
& \left|\int_{\mathbb{R}^{d}} u_{\lambda}(t, x) \psi(x) d x-\int_{\mathbb{R}^{d}} u_{\lambda}(0, x) \psi(x) d x\right| \\
& \quad \leq\left\|D^{2} \psi\right\|_{L^{\infty}\left(\mathbb{R}^{d}\right)} \int_{0}^{t} \int_{\mathbb{R}^{d}} u_{\lambda}(s, x) d x d s+\lambda^{d(1-q)+1}\|D \psi\|_{L^{\infty}\left(\mathbb{R}^{d}\right)} \int_{0}^{t} \int_{\mathbb{R}^{d}} u_{\lambda}^{q}(s, x) d x d s \\
& \quad \lesssim t\left\|D^{2} \psi\right\|_{L^{\infty}\left(\mathbb{R}^{d}\right)}+t^{1 / 2}\|D \psi\|_{L^{\infty}\left(\mathbb{R}^{d}\right)} .
\end{aligned}
$$


Letting $\lambda \rightarrow \infty$ and using that for any $t>0, u_{\lambda}(t) \rightarrow U(t)$ in $L^{2}\left(\mathbb{R}^{d}\right)$ we get

$$
\left|\int_{\mathbb{R}^{d}} U(t, x) \psi(x) d x-m \psi(0)\right| \lesssim t\left\|D^{2} \psi\right\|_{L^{\infty}\left(\mathbb{R}^{d}\right)}+t^{1 / 2}\|D \psi\|_{L^{\infty}\left(\mathbb{R}^{d}\right)}, \forall t>0,
$$

where $m$ is the mass of the initial data $\varphi$. This shows that for any $\psi \in C_{c}^{2}\left(\mathbb{R}^{d}\right)$

$$
\lim _{t \downarrow 0} \int_{\mathbb{R}^{d}} U(t, x) \psi(x) d x=m \psi(0) .
$$

We want to show that this is true for any smooth bounded function $\psi$ and then $U(t) \rightarrow m \delta_{0}$ in the weak sense of nonnegative measures in $\mathbb{R}^{d}$.

Let us now choose $\psi$ a bounded smooth function. For any $\varepsilon>0$ we choose $\psi_{\varepsilon} \in C_{c}^{2}\left(\mathbb{R}^{d}\right)$ such that $\left\|\psi-\psi_{\varepsilon}\right\|_{L^{\infty}\left(\mathbb{R}^{d}\right)} \leq \varepsilon$. Then

$$
\begin{aligned}
& \left|\int_{\mathbb{R}^{d}} U(t, x) \psi(x) d x-m \psi(0)\right| \\
& \quad \leq\left|\int_{\mathbb{R}^{d}} U(t, x)\left(\psi(x)-\psi_{\varepsilon}(x)\right) d x\right|+m\left|\psi(0)-\psi_{\varepsilon}(0)\right|+\left|\int_{\mathbb{R}^{d}} U(t, x) \psi_{\varepsilon}(x) d x-m \psi_{\varepsilon}(0)\right| \\
& \quad \leq 2 \varepsilon m+\left\|\psi_{\varepsilon}\right\|_{W^{2, \infty}\left(\mathbb{R}^{d}\right)}\left(t+t^{1 / 2}\right) .
\end{aligned}
$$

Thus there exists $t_{0}=t_{0}(\varepsilon)$ such that for all $t \in\left(0, t_{0}\right)$ the following holds

$$
\left|\int_{\mathbb{R}^{d}} U(t, x) \psi(x) d x-m \psi(0)\right| \leq 4 \varepsilon m .
$$

This shows that $U(t)$ goes to $m \delta_{0}$ as $t \rightarrow 0$ in the sense of measures.

In conclusion the limit point $U$ satisfies $U \in L_{l o c}^{\infty}\left((0, \infty), L^{1}\left(\mathbb{R}^{d}\right)\right) \cap L_{l o c}^{2}\left((0, \infty), H^{1}\left(\mathbb{R}^{d}\right)\right)$. When $q>1+1 / d$ or $B=0_{1, d}, U$ is a solution of the heat equation $U_{t}=A \Delta U$ with $m \delta_{0}$ initial data. When $q=1+1 / d$ and $B \neq 0_{1, d}, U$ is a solution of the equation:

$$
\left\{\begin{array}{l}
U_{t}=A \Delta U-B \cdot \nabla U^{q}, \quad x \in \mathbb{R}^{d}, t>0, \\
U(0)=m \delta_{0} .
\end{array}\right.
$$

Since for any $\tau>0$ we have $U(\tau) \in L^{1}\left(\mathbb{R}^{d}\right)$ classical results on parabolic equations show that for any $\tau>0$

$$
U \in C\left((\tau, \infty), L^{1}\left(\mathbb{R}^{d}\right)\right) \cap L^{\infty}\left((\tau, \infty) \times \mathbb{R}^{d}\right) .
$$

Using the fact that the heat system as well as system (3.24) have a unique solution (see [9], [10] for complete details) then the full sequence $\left\{u_{\lambda}\right\}$, not only a subsequence, converges to $U$.

Step IV. The asymptotic behavior. We recall that from Step II we have

$$
\left\|u_{\lambda}(1)-U(1)\right\|_{L^{1}\left(\mathbb{R}^{d}\right)} \rightarrow 0 \quad \text { as } \lambda \rightarrow \infty .
$$

After setting $t=\lambda^{2}$ and using the self-similar form of $U(t, x)$

$$
U(t, x)=t^{-d / 2} U\left(1, x t^{-1 / 2}\right)=t^{-d / 2} f_{m}\left(x t^{-1 / 2}\right)
$$


we obtain that solution $u$ of system (1.1) satisfies

$$
\lim _{t \rightarrow \infty}\|u(t)-U(t)\|_{L^{1}\left(\mathbb{R}^{d}\right)}=0 .
$$

This is exactly (1.6) in the case $p=1$. The general case, $1 \leq p<\infty$, follows immediately since

$$
\begin{aligned}
\|u(t)-U(t)\|_{L^{p}\left(\mathbb{R}^{d}\right)} & \lesssim\|u(t)-U(t)\|_{L^{1}\left(\mathbb{R}^{d}\right)}^{\frac{1}{2-1}}\left(\|u(t)\|_{L^{2 p}\left(\mathbb{R}^{d}\right)}+\|U(t)\|_{L^{2 p}\left(\mathbb{R}^{d}\right)}\right)^{\frac{2 p-2}{2 p-1}} \\
& \leq\|u(t)-U(t)\|_{L^{1}\left(\mathbb{R}^{d}\right)}^{\frac{1}{2 p-1}} t^{-\frac{d}{2}\left(1-\frac{1}{p}\right)}=o\left(t^{-\frac{d}{2}\left(1-\frac{1}{p}\right)}\right) .
\end{aligned}
$$

The proof of Theorem 1.2 is now completed.

\section{Acknowledgements.}

L. Ignat was partially supported by Grant PN-II-ID-PCE-2011-3-0075 of the Romanian National Authority for Scientific Research, CNCS - UEFISCDI.

T. Ignat was supported by Grant PN-II-ID-PCE-2012-4-0021 of the Romanian National Authority for Scientific Research, CNCS - UEFISCDI and by a doctoral fellowship offered by IMAR.

D. Stancu-Dumitru was partially supported by grant CNCS-UEFISCDI PN-II-ID-PCE2011-3-0075 Analysis, Control and Numerical Approximations of Partial Differential Equations.

Parts of this paper have been done during the visit of the authors at BCAM-Basque Center for Applied Mathematics, Bilbao, Spain. The authors thank the center for hospitality and support. The authors thank J.L. Vazquez, J.D. Rossi and M. Escobedo for fruitful discussions.

\section{REFERENCES}

[1] J. Aguirre, M. Escobedo, and E. Zuazua. Self-similar solutions of a convection diffusion equation and related semilinear elliptic problems. Comm. Partial Differential Equations, 15(2):139-157, 1990.

[2] Fuensanta Andreu-Vaillo, José M. Mazón, Julio D. Rossi, and J. Julián Toledo-Melero. Nonlocal diffusion problems. Mathematical Surveys and Monographs 165. Providence, RI: American Mathematical Society (AMS); Madrid: Real Sociedad Matemática Española. xv, 256 p. \$ $82.00,2010$.

[3] Jean Bourgain, Haim Brezis, and Petru Mironescu. Another look at Sobolev spaces. Menaldi, José Luis (ed.) et al., Optimal control and partial differential equations. In honour of Professor Alain Bensoussan's 60th birthday. Proceedings of the conference, Paris, France, December 4, 2000. Amsterdam: IOS Press; Tokyo: Ohmsha. 439-455 (2001)., 2001.

[4] A. Carpio. Large time behaviour in convection-diffusion equations. Ann. Scuola Norm. Sup. Pisa Cl. Sci. (4), 23(3):551-574, 1996.

[5] E. Chasseigne, M. Chaves, and J. D. Rossi. Asymptotic behavior for nonlocal diffusion equations. J. Math. Pures Appl. (9), 86(3):271-291, 2006.

[6] Qiang Du, James R. Kamm, R. B. Lehoucq, and Michael L. Parks. A new approach for a nonlocal, nonlinear conservation law. SIAM J. Appl. Math., 72(1):464-487, 2012.

[7] Renjun Duan, Lizhi Ruan, and Changjiang Zhu. Optimal decay rates to conservation laws with diffusion-type terms of regularity-gain and regularity-loss. Math. Models Methods Appl. Sci., 22(7):1250012, 39, 2012.

[8] M. Escobedo and E. Zuazua. Large time behavior for convection-diffusion equations in $\mathbf{R}^{N}$. J. Funct. Anal., 100(1):119-161, 1991. 
[9] Miguel Escobedo, Juan Luis Vázquez, and Enrike Zuazua. Asymptotic behaviour and source-type solutions for a diffusion-convection equation. Arch. Rational Mech. Anal., 124(1):43-65, 1993.

[10] Miguel Escobedo, Juan Luis Vázquez, and Enrike Zuazua. A diffusion-convection equation in several space dimensions. Indiana Univ. Math. J., 42(4):1413-1440, 1993.

[11] Loukas Grafakos. Classical Fourier analysis, volume 249 of Graduate Texts in Mathematics. Springer, New York, second edition, 2008.

[12] Liviu I. Ignat and Ademir F. Pazoto. Large time behaviour for a nonlocal diffusion - convection equation related with gas dynamics. Discrete Contin. Dyn. Syst., 34(9):3575-3589, 2014.

[13] Liviu I. Ignat and Alejandro Pozo. A splitting method for the augmented burgers equation. in preparation.

[14] Liviu I. Ignat and Julio D. Rossi. A nonlocal convection-diffusion equation. J. Funct. Anal., 251(2):399-437, 2007.

[15] Liviu I. Ignat and Julio D. Rossi. Refined asymptotic expansions for nonlocal diffusion equations. $J$. Evol. Equ., 8(4):617-629, 2008.

[16] Liviu I. Ignat and Julio D. Rossi. Decay estimates for nonlocal problems via energy methods. J. Math. Pures Appl. (9), 92(2):163-187, 2009.

[17] Liviu I. Ignat and Julio D. Rossi. Asymptotic expansions for nonlocal diffusion equations in $L^{q}$-norms for $1 \leq q \leq 2$. J. Math. Anal. Appl., 362(1):190-199, 2010.

[18] Tatiana I. Ignat. Asymptotics for nonlocal evolution problems by scaling arguments. Differ. Equ. Appl., 5(4):613-626, 2013.

[19] Tatiana I. Ignat. Analytic and stochastic methods for nonlocal operators, PhD Thesis. IMAR, Bucharest, Romania, in preparation.

[20] Grzegorz Karch and Kanako Suzuki. Spikes and diffusion waves in a one-dimensional model of chemotaxis. Nonlinearity, 23(12):3119-3137, 2010.

[21] S. N. Kružkov. First order quasilinear equations with several independent variables. Mat. Sb. (N.S.), 81 (123):228-255, 1970.

[22] Philippe Laurençot. Asymptotic self-similarity for a simplified model for radiating gases. Asymptot. Anal., 42(3-4):251-262, 2005.

[23] P.-L. Lions, B. Perthame, and E. Tadmor. A kinetic formulation of multidimensional scalar conservation laws and related equations. J. Amer. Math. Soc., 7(1):169-191, 1994.

[24] Augusto C. Ponce. An estimate in the spirit of Poincaré's inequality. J. Eur. Math. Soc. (JEMS), $6(1): 1-15,2004$.

[25] M. Schonbek. Decay of solutions to parabolic conservation laws. Comm. Partial Differential Equations, $5(5): 449-473,1980$.

[26] M. Schonbek. Uniform decay rates for parabolic conservation laws. Nonlinear Anal., 10(9):943-956, 1986.

[27] M. Schonbek. The Fourier splitting method. In Advances in geometric analysis and continuum mechanics (Stanford, CA, 1993), pages 269-274. Int. Press, Cambridge, MA, 1995.

[28] Denis Serre. $L^{1}$-stability of nonlinear waves in scalar conservation laws. In Evolutionary equations. Vol. I, Handb. Differ. Equ., pages 473-553. North-Holland, Amsterdam, 2004.

[29] Jacques Simon. Compact sets in the space $L^{p}(0, T ; B)$. Ann. Mat. Pura Appl. (4), 146:65-96, 1987.

[30] Joana Terra and Noemi Wolanski. Asymptotic behavior for a nonlocal diffusion equation with absorption and nonintegrable initial data. The supercritical case. Proc. Amer. Math. Soc., 139(4):1421-1432, 2011.

[31] Joana Terra and Noemi Wolanski. Large time behavior for a nonlocal diffusion equation with absorption and bounded initial data. Discrete Contin. Dyn. Syst., 31(2):581-605, 2011.

[32] Juan Luis Vázquez. Asymptotic beahviour for the porous medium equation posed in the whole space. J. Evol. Equ., 3(1):67-118, 2003. Dedicated to Philippe Bénilan. 
L. I. IGNAT

Institute of Mathematics "Simion Stollow" of the Romanian Academy, 21 Calea Grivitei Street, 010702 Bucharest, Romania

AND

Faculty of Mathematics and Computer Science, University of Bucharest, 14 Academiei Street, 010014 Bucharest, Romania.

E-mail address: liviu.ignat@gmail.com

Web page: http://www.imar.ro/ lignat

T. I. IGNAT

Institute of Mathematics "Simion Stoilow" of the Romanian Academy, 21 Calea Grivitei Street, 010702 Bucharest, Romania

E-mail address: tatiana.ignat@gmail.com

Denisa Stancu-Dumitru

Institute of Mathematics "Simion Stollow" of the Romanian Academy, 21 Calea Grivitei Street, 010702 Bucharest, Romania

E-mail address: denisa.stancu@yahoo.com 